\title{
Hierarchical Generalized Additive Models in ecology: an introduction with mgcv
}

\author{
Eric J Pedersen ${ }^{\text {Corresp., } 1,2}$, David L. Miller ${ }^{3,4}$, Gavin L. Simpson ${ }^{5,6}$, Noam Ross ${ }^{7}$ \\ ${ }^{1}$ Northwest Atlantic Fisheries Center, Fisheries and Oceans Canada, St. John's, Newfoundland and Labrador, Canada \\ 2 Department of Biology, Memorial University of Newfoundland, St. John's, Newfoundland and Labrador, Canada \\ 3 Centre for Research into Ecological and Environmental Modelling, University of St Andrews, St Andrews, United Kingdom \\ 4 School of Mathematics and Statistics, University of St Andrews, St. Andrews, United Kingdom \\ 5 Institute of Environmental Change and Society, University of Regina, Regina, Saskatchewan, Canada \\ 6 Department of Biology, University of Regina, Regina, Saskatchewan, Canada \\ 7 Ecohealth Alliance, New York, New York, United States \\ Corresponding Author: Eric J Pedersen \\ Email address: eric.pedersen@dfo-mpo.gc.ca
}

In this paper, we discuss an extension to two popular approaches to modelling complex structures in ecological data: the generalized additive model (GAM) and the hierarchical model (HGLM). The hierarchical GAM (HGAM), allows modelling of nonlinear functional relationships between covariates and outcomes where the shape of the function itself varies between different grouping levels. We describe the theoretical connection between HGAMs, HGLMs and GAMs, explain how to model different assumptions about the degree of inter-group variability in functional response, and show how HGAMs can be readily fitted using existing GAM software, the mgcr package in R. We also discuss computational and statistical issues with fitting these models, and demonstrate how to fit HGAMs on example data. All code and data used to generate this paper are available at: github.com/ericpedersen/mixed-effect-gams. 


\section{- Hierarchical Generalized Additive Models in ecology: an introduction with mgcv}

${ }_{3}$ Eric J. Pedersen ${ }^{1,2,}$, David L. Miller ${ }^{3,4}$, Gavin L. Simpson ${ }^{5,6}$, and Noam Ross ${ }^{7}$

${ }_{4} \quad{ }^{1}$ Northwest Atlantic Fisheries Center, Fisheries and Oceans Canada, St.

$5 \quad$ John's, NL, Canada

$6{ }^{2}$ Department of Biology, Memorial University of Newfoundland, St. John's,

7

8
Newfoundland and Labrador, Canada

${ }^{3}$ Centre for Research into Ecological and Environmental Modelling, University of St Andrews, St Andrews, UK

${ }^{4}$ School of Mathematics and Statistics, University of St Andrews, St Andrews, UK Regina, SK, Canada ${ }^{6}$ Department of Biology, University of Regina, Regina, SK, Canada

${ }^{7}$ Ecohealth Alliance, New York, NY, USA

${ }^{*}$ Corresponding author. Email: eric.j.pedersen@gmail.com

\section{Abstract}

In this paper, we discuss an extension to two popular approaches to modelling complex structures in ecological data: the generalized additive model (GAM) and the hierarchical model (HGLM). The hierarchical GAM (HGAM), allows modelling of nonlinear functional relationships between covariates and outcomes where the shape of the function itself varies between different grouping levels. We describe the theoretical connection between HGAMs, HGLMs and GAMs, explain how to model different assumptions about the degree of intergroup variability in functional response, and show how HGAMs can be readily fitted using existing GAM software, the mgcv package in R. We also discuss computational and statistical issues with fitting these models, and demonstrate how to fit HGAMs on example data. All code and data used to generate this paper are available at: github.com/eric-pedersen/mixedeffect-gams. 
${ }_{29}$ I: Introduction

Two of the most popular and powerful modelling techniques currently in use by ecologists are generalized additive models (GAMs; Wood, 2017a) for modelling flexible regression functions, and generalized linear mixed models ("hierarchical generalized linear models" (HGLMs) or simply "hierarchical models"; Bolker et al., 2009; Gelman et al., 2013) for modelling between-group variability in regression relationships.

At first glance, GAMs and HGLMs are very different tools used to solve different problems. GAMs are used to estimate smooth functional relationships between predictor variables and the response. HGLMs, on the other hand, are used to estimate linear relationships between predictor variables and response (although nonlinear relationships can also be modeled through quadratic terms or other transformations of the predictor variables), but impose a structure where predictors are organized into groups (often referred to as "blocks") and the relationships between predictor and response may vary across groups. Either the slope or intercept, or both, may be subject to grouping. A typical example of HGLM use might be to include site-specific effects in a model of population counts, or to model individual level heterogeneity in a study with repeated observations of multiple individuals.

However, the connection between HGLMs and GAMs is quite deep, both conceptually and mathematically (Verbyla et al., 1999). HGLMs and GAMs fit highly variable models by "pooling" parameter estimates towards one another, by penalizing squared deviations from some simpler model. In an HGLM, this occurs as group-level effects are pulled towards global effects (penalizing the squared differences between each group-level parameter estimate and the global effect). In a GAM, this occurs via the enforcement of a smoothness criterion on the variability of a functional relationship, pulling parameters towards some function that is assumed to be totally smooth (such as a straight line) by penalizing squared deviations from that totally smooth function.

Given this connection, a natural extension to the standard GAM framework is to allow smooth functional relationships between predictor and response to vary between groups, but in such a way that the different functions are in some sense pooled toward a common shape. We often want to know both how functional relationships vary between groups, and if a relationship holds across groups. We will refer to this type of model as a hierarchical GAM, or HGAM.

There are many potential uses for HGAMs. For example, we can use them to estimate how the maximum size of different fish species varies along a common temperature gradient (Fig. 1). Each species will typically have its own response function, but since the species overlap in range, they should have similar responses over at least some of the temperature gradient; Figure 1 shows all three species reach their largest maximum sizes in the centre of the temperature gradient. Estimating a separate function for each species throws away a lot of shared information and could result in highly noisy function estimates if there were only a few data points for each species. Estimating a single average relationship could result in a function that did not predict any specific group well. In our example, using a single global temperature-size relationship (Fig. 1, solid line) would miss that the three species have distinct temperature optima, and that the orange species is significantly smaller at all temperatures than the other two (Fig. 1). We prefer a hierarchical model that includes a 


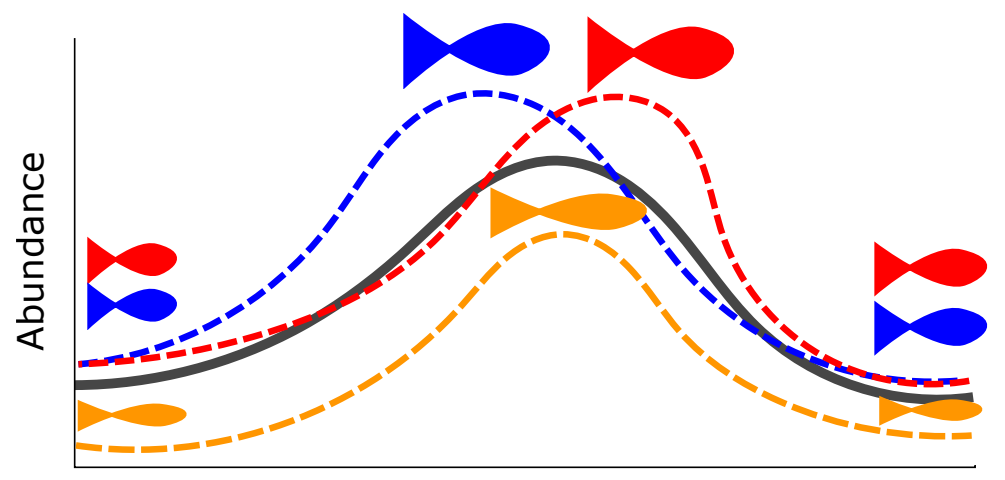

Temperature

Figure 1: Hypothetical example of functional variability between different group levels. Each dashed line indicates how the abundance for different species of fish in a community might vary as a function of average water temperature. The orange species shows lower abundance at all temperatures, and the red and blue species differ at which temperature they can achieve the maximum possible size. However, all three curves are similiarly smooth and peak close to one another relative to the entire range of tested temperatures. The solid black line represents an 'average abundance curve', representing the mean abundance across species in the sample.

global temperature-size curve plus species-specific curves that were penalized to be close to the mean function.

This paper discusses several approaches to group-level smoothing, and corresponding trade-offs. We focus on fitting HGAMs with the popular mgcv package (Wood, 2011) for the R statistical programming language (R Core Team, 2018), which allows for a variety of HGAM model structures and fitting strategies. We discuss options available to the modeller and practical and theoretical reasons for choosing them. We demonstrate the different approaches across a range of case studies.

This paper is divided into five sections. Part II is a brief review of how GAMs work and their relation to hierarchical models. In part III, we discuss different HGAM formulations, what assumptions each model makes about how information is shared between groups, and the different ways of specifying these models in mgcv. In part IV, we work through example analyses using this approach, to demonstrate the modelling process and how HGAMs can be incorporated into the ecologist's quantitative toolbox. Finally, in part V, we discuss some of the computational and statistical issues involved in fitting HGAMs in mgcv. We have also included all the code needed to reproduce the results in this manuscript in supplemental code (online), and on the GitHub repository associated with this paper: github.com/ericpedersen/mixed-effect-gams.

\section{II: A review of Generalized Additive Models}

The generalized linear model (GLM; McCullagh \& Nelder, 1989) relates the mean of a response $(y)$ to a linear combination of explanatory variables. The response is assumed to be 
conditionally distributed according to some exponential family distribution (e.g., binomial, Poisson or Gamma distributions for trial, count or strictly positive real responses, respectively). The generalized additive model (GAM; Hastie \& Tibshirani, 1990; Ruppert, Wand \& Carroll, 2003; Wood, 2017a) allows the relationships between the explanatory variables (henceforth covariates) and the response to be described by smooth curves (usually splines (de Boor, 1978), but potentially other structures). In general we have models of the form:

$$
\mathbb{E}(Y)=g^{-1}\left(\beta_{0}+\sum_{j=1}^{J} f_{j}\left(x_{j}\right)\right),
$$

where $\mathbb{E}(Y)$ is the expected value of the response $Y$ (with an appropriate distribution and link function $g$ ), $f_{j}$ is a smooth function of the covariate $x_{j}, \beta_{0}$ is an intercept term and $g^{-1}$ is the inverse link function. Hereafter, we will refer to these smooth functions as smoothers. In the example equation above, there are $J$ smoothers and each is a function of only one covariate, though it is possible to construct smoothers of multiple variables.

Each smoother $f_{j}$ is represented by a sum of $K$ simpler, fixed basis functions $\left(b_{j, k}\right)$ multiplied by corresponding coefficients $\left(\beta_{j, k}\right)$, which need to be estimated:

$$
f_{j}\left(x_{j}\right)=\sum_{k=1}^{K} \beta_{j, k} b_{j, k}\left(x_{j}\right) .
$$

$K$, referred to as "basis size", "basis complexity" or "basis richness", determines the maximum complexity of each smoother.

It would seem that large basis size could lead to overfitting, but this is counteracted by a smoothing penalty that influences basis function coefficients so as to prevent excess wiggliness and ensure appropriate complexity of each smoother. For each smoother, one (or more) penalty matrices $(\mathbf{S})$, specific to the form of the basis functions, is pre- and post-multiplied by the parameter vector $\boldsymbol{\beta}$ to calculate the penalty $\left(\boldsymbol{\beta}^{T} \mathbf{S} \boldsymbol{\beta}\right)$. A penalty term is then subtracted from the model log-likelihood $L$, controlling the trade-off via a smoothing parameter $(\lambda)$. The penalized log-likelihood used to fit the model is thus:

$$
L-\boldsymbol{\lambda} \boldsymbol{\beta}^{T} \mathbf{S} \boldsymbol{\beta}
$$

Figure 2 shows an example of how different choices of the smoothing parameter $(\lambda)$ affect the shape of the resulting smoother. Data (points) were generated from the blue function and noise added to them. In Fig. 2a, $\lambda$ was selected using Restricted Maximum Likelihood (REML) to give a good fit to the data. In Fig. $2 \mathrm{~b}, \lambda$ was set to zero so the penalty has no effect and the function interpolates the data. Figure 2c shows when $\lambda$ is set to a very large value, so the penalty removes all terms that have any wiggliness, giving a straight line.

To measure the complexity of a penalized smooth terms we use the effective degrees of freedom $(\mathrm{EDF})$, which at a maximum is the number of coefficients to be estimated in the model, minus any constraints. The EDF can take non-integer values and larger values indicate more wiggly terms (see Wood (2017a, Section 6.1.2) for further details). The number of basis functions, $K$ sets a maximum for the EDF, as a smoother cannot have more than $K$ EDF. When the EDF 

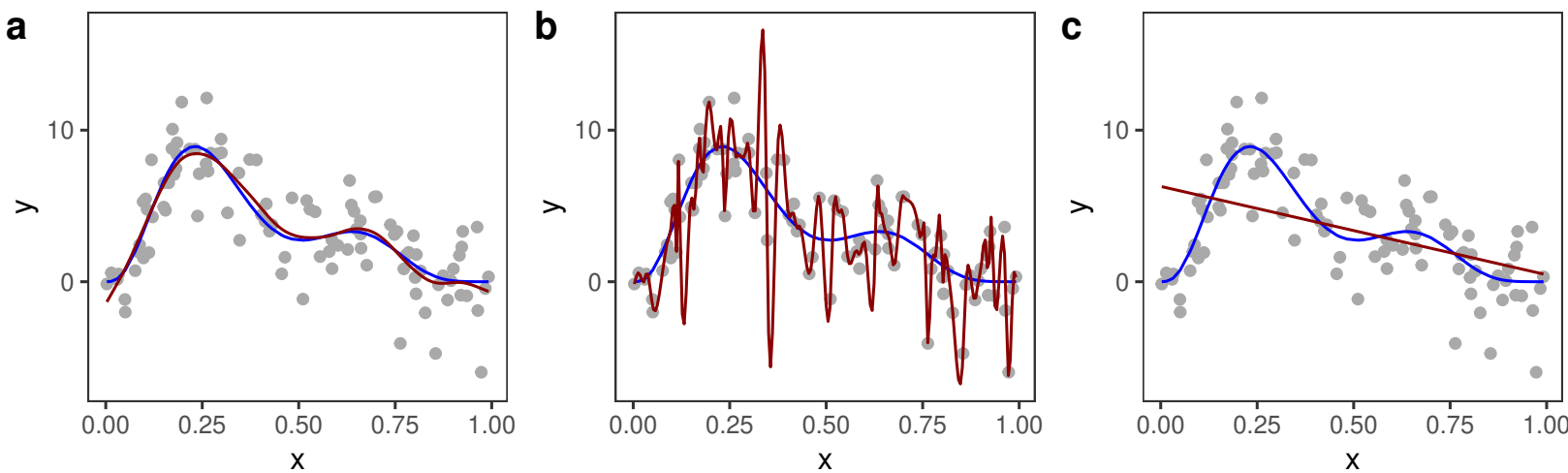

Figure 2: Effect of different choices of smoothing parameter $(\lambda)$ on the shape of the resulting smoother (red lines). a) $\lambda$ estimated using REML; b) $\lambda$ set to zero (no smoothing); c) $\lambda$ is set to a very large value. The blue line in each panel is the known model used to simulate the data.

is well below $K$, increasing $K$ generally has very little effect on the shape of the function. In general, $K$ should be set large enough to allow for potential variation in the smoother while still staying low enough to keep computation time low (see section $\mathrm{V}$ for more on this). In mgcv, the function mgcv: : check. gam can be used to determine if $k$ has been set too low.

Random effects are also "smooths" in this framework. In this case, the penalty matrix is the inverse of the correlation matrix of the basis function coefficients (Kimeldorf \& Wahba, 1970; Wood, 2017a). For a simple single-level random effect to account for variation in group means (intercepts) there will be one basis function for each level of the grouping variable. The basis function takes a value of 1 for any observation in that group and 0 for any observation not in the group. The penalty matrix for these terms is a $g$ by $g$ identity matrix, where $g$ is the number of groups. This means that each group-level coefficient will be penalized in proportion to its squared deviation from zero. This is equivalent to how random effects are estimated in standard mixed effect models. The penalty term is proportional to the inverse of the variance of the fixed effect estimated by standard hierarchical model software (Verbyla et al., 1999).

This connection between random effects and splines extends beyond the varying-intercept case. Any single-penalty basis-function representation of a smooth can be transformed so that it can be represented as a combination of a random effect with an associated variance, and possibly one or more fixed effects. See Verbyla et al. (1999) or Wood, Scheipl \& Faraway (2013) for a more detailed discussion on the connections between these approaches.

\section{Basis types and penalty matrices}

The range of smoothers are useful for contrasting needs and have different associated penalty matrices for their basis function coefficients. In the examples in this paper, we will use three types of smoothers: thin plate regression splines, cyclic cubic regression splines, and random effects. 
Thin plate regression splines (TPRS; Wood, 2003) are a general purpose spline basis which can be used for problems in any number of dimensions, provided one can assume that the amount of smoothing in any of the covariates is the same (so called isotropy or rotational invariance). TPRS, like many splines, use a penalty matrix made up of terms based on the the integral of the squared derivatives of basis functions across their range (see Wood (2017a) page 216 for details on this penalty). Models that overfit the data will tend to have large derivatives, so this penalization reduces wiggliness. We will refer to the order of penalized derivatives by $m$. Typically, TPRS are second-order $(m=2)$, meaning that the penalty is proportionate to the integral of the squared second derivative. However, TPRS may be of lower order ( $m=1$, penalizing squared first derivatives), or higher order ( $m>2$, penalizing squared higher order derivatives). We will see in section III how lower-order TPRS smoothers are useful in fitting HGAMs. Example basis functions and penalty matrix $\mathbf{S}$ for a $m=2$ TPRS with six basis functions for evenly spaced data are shown in Fig. 3.

Cyclic cubic regression splines (CRS) are another smoother that penalizes the squared second derivative of the smooth across the function. In cyclic CRS the start and end of the smoother are constrained to match in value and first derivative. These are useful for fitting models with cyclic components such as seasonal effects. We will use these smoothers to demonstrate how to fit HGAMs to cyclic data.

\section{Smoothing penalties vs. shrinkage penalties}

Penalties can have two effects on how well a model fits: they can penalize how wiggly a given term is (smoothing) and they can penalize the absolute size of the function (shrinkage). The penalty can only affect the components of the smoother that have derivatives (the range space), not the other parts (the null space). For 1-dimensional TPRS (when $m=2$ ), this means that there is a linear term (F5) left in the model, even when the penalty is in full force (as $\lambda \rightarrow \infty$ ), as shown in Fig. 3. (This is also why Fig. 2c shows a linear, rather than flat, fit to the data). The random effects smoother we discussed earlier is an example of a pure shrinkage penalty; it penalizes all deviations away from zero, no matter the pattern of those deviations. This will be useful later in section III, where we use random effect smoothers as one of the components of a HGAM.

\section{Interactions between smooth terms}

It is also possible to create interactions between covariates with different smoothers (or degrees of smoothness) assumed for each covariate, using tensor products. For instance, if one wanted to estimate the interacting effects of temperature and time (in seconds) on some outcome, it would not make sense to use a two-dimensional TPRS smoother, as that would assume that a one degree change in temperature would equate to a one second change in time. Instead, a tensor product allows us to create a new set of basis functions that allow for each marginal function (here temperature and time) to have its own marginal smoothness penalty. A different basis can be used in each marginal smooth, as required for the data at hand.

There are two approaches used in $\mathbf{m g c v}$ for generating tensor products. The first approach (Wood, 2006a) essentially creates an interaction of each pair of basis functions for each 
a

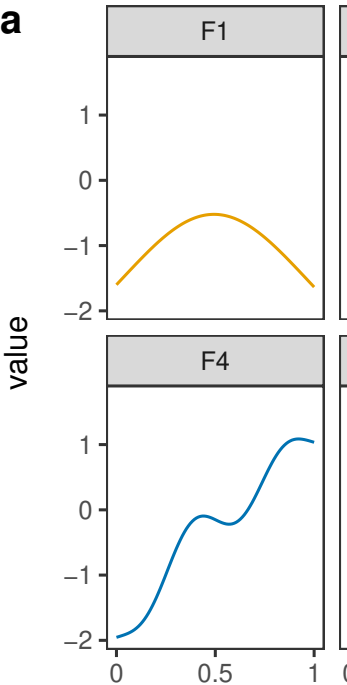

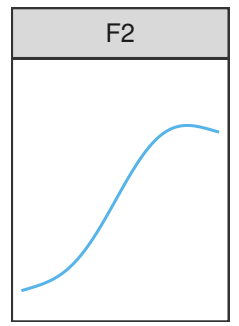

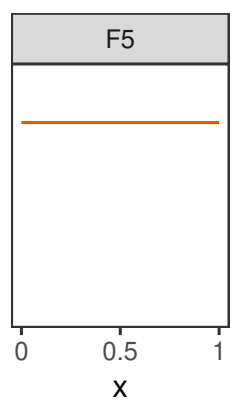

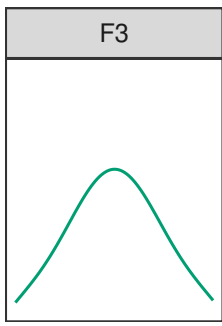

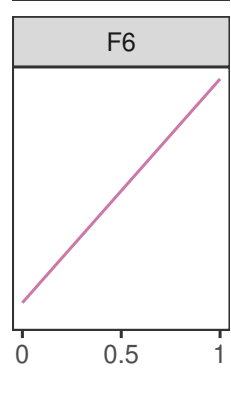

b

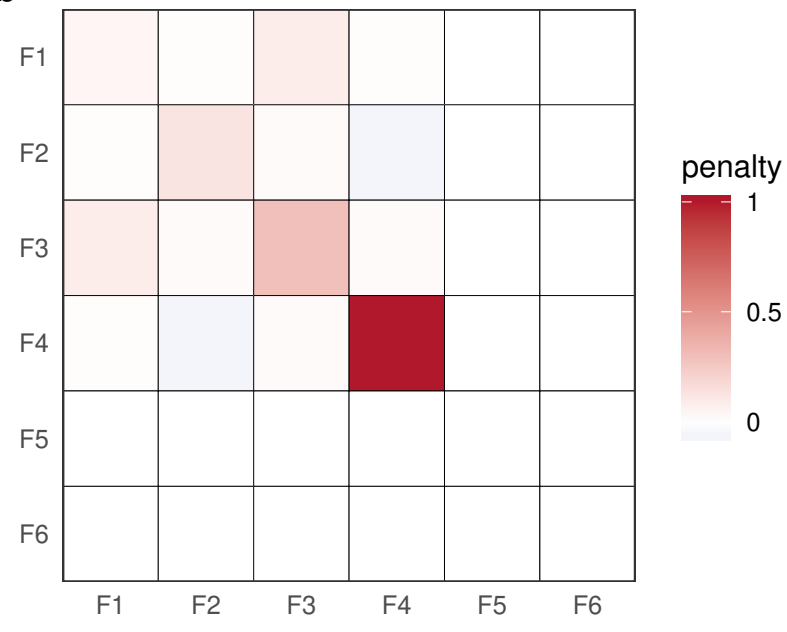

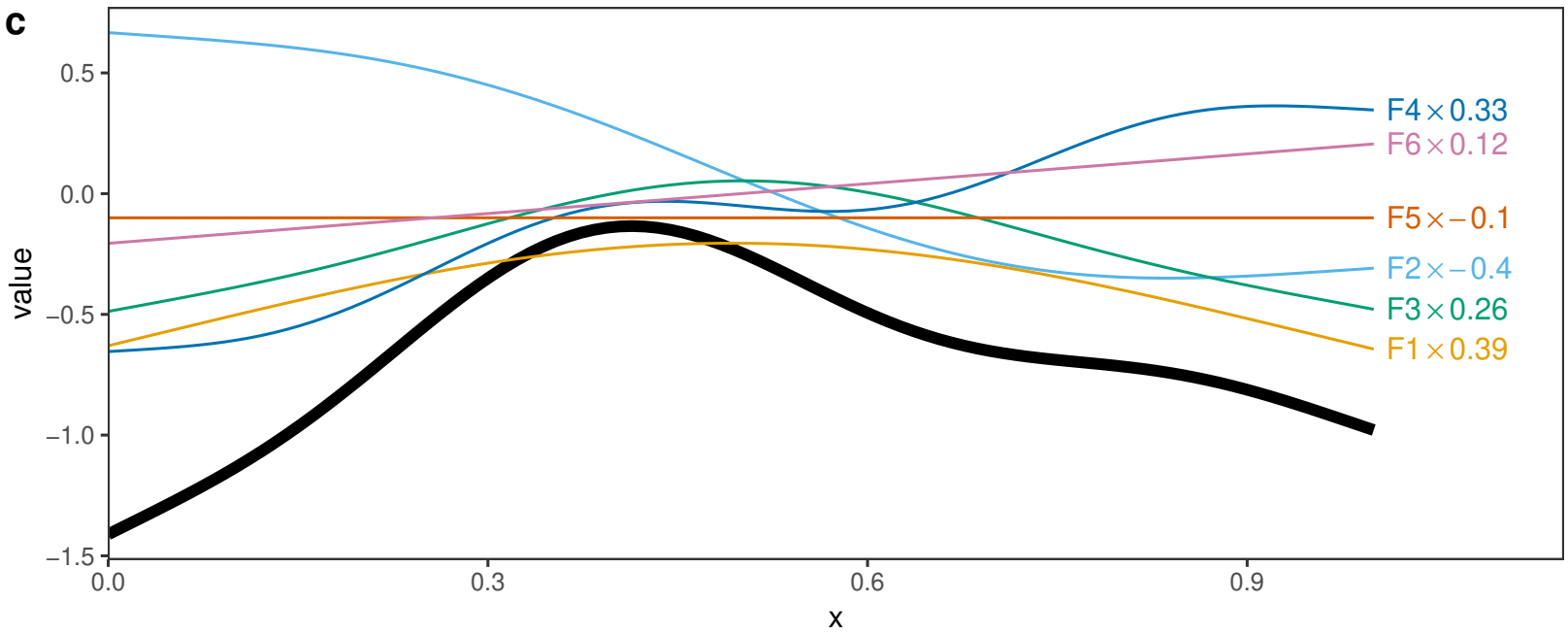

Figure 3: a) Examples of the basis functions associated with a six basis function thin plate regression spline (TPRS, $\mathrm{m}=2$ ), calculated for data, $x$, spread evenly between $x=0$ and $x=1$. Each line represents a single basis function. b) The smoothing penalty matrix for the thin plate smoother. Red entries indicate positive values and blue indicate negative values. For example, functions F3 and F4 would have the greatest proportionate effect on the total penalty (as they have the largest values on the diagonal), whereas function F5 and F6 would not contribute to the wiggliness penalty at all (all the values in the 5th and 6th row and column of the penalty matrix are zero). This means these functions are in the null space of the penalty matrix, and are treated as completely smooth. c) An example of how the basis functions add up to create a single smooth function. Thin coloured lines represent each basis function multiplied by a coefficient, and the solid black line is the sum of those basis functions. 
marginal term, and a penalty for each marginal term that penalizes the average wiggliness in that term; in mgcv, these are created using the te() function. The second approach (Wood, Scheipl \& Faraway, 2013) separates each penalty into penalized (range space) and unpenalized components (null space; components that don't have derivatives, such as intercept and linear terms in a one-dimensional cubic spline). This approach creates new basis functions and penalties for all pair-wise combinations of penalized and unpenalized components between all pairs of marginal bases; in mgcv, these are created using the t2() function. The advantage of the first method is that it requires fewer smoothing parameters, so is faster to estimate in most cases. The advantage of the second method is that the tensor products created this way only have a single penalty associated with each marginal basis (unlike the te() approach, where each penalty applies to all basis functions), so it can be fitted using standard mixed effect software such as lme4 (Bates et al., 2015).

\section{Comparison to hierarchical linear models}

Hierarchical generalized linear models (Gelman, 2006; HGLMs; also referred to as generalized linear mixed effect models, multilevel models etc; e.g., Bolker et al., 2009) are an extension of regression modelling that allows the inclusion of terms in the model that account for structure in the data - the structure is usually of the form of a nesting of the observations. For example, in an empirical study, individuals may be nested within sample sites, sites are nested within forests, and forests within provinces. The depth of the nesting is limited by the fitting procedure and number of parameters to estimate.

HGLMs are a highly flexible way to think about grouping in ecological data; the groupings used in models often refer to the spatial or temporal scale of the data (McMahon \& Diez, 2007) though can be based on any useful grouping.

We would like to be able to think about the groupings in our data in a similar way, even when the covariates in our model are related to the response in a smooth way. The next section investigates the extension of the smoothers we showed above to the case where observations are grouped and we model group-level smoothers.

\section{III: What are hierarchical GAMs?}

\section{What do we mean by hierarchical smoothers?}

In this section, we will describe how to model inter-group variability using smooth curves and how to fit these models using mgcv. All models were fitted using mgcv version 1.8-26 (Wood, 2011). Model structure is key in this framework, so we start with three model choices:

1. Should each group have its own smoother, or will a common smoother suffice?

2. Do all of the group-specific smoothers have the same wiggliness, or should each group have its own smoothing parameter?

3. Will the smoothers for each group have a similar shape to one another - a shared global smoother?

These three choices result in five possible models (Fig. 4): 


\footnotetext{
${ }^{1}$ Note that mgcv requires that grouping or categorical variables be coded as factors in $\mathrm{R}$; it will raise an
} only has a Global smoother.

2. A global smoother plus group-level smoothers that have the same wiggliness. We will refer to this as model $G S$ (for Global smoother with individual effects that have a Shared penalty)

3. A global smoother plus group-level smoothers with differing wiggliness. We will refer to this as model GI (for Global smoother with individual effects that have Individual penalties)

4. Group-specific smoothers without a global smoother, but with all smoothers having the same wiggliness. We will refer to this as model $S$.

5. Group-specific smoothers with different wiggliness. We will refer to this as model $I$.

It is important to note that "similar wiggliness" and "similar shape" are two distinct concepts; functions can have very similar wiggliness but very different shapes. Wiggliness measures how quickly a function changes across its range, and it is easy to construct two functions that differ in shape but have the same wiggliness. For this paper, we consider two functions to have similar shape if the average squared distance between the functions is small (assuming the functions have been scaled to have a mean value of zero across their ranges). This definition is somewhat restricted; for instance, a cyclic function would not be considered to have the same shape as a phase-shifted version of the same function, nor would two normal distributions with the same mean but different standard deviations. The benefit of this definition of shape, however, is that it is straightforward to translate into penalties akin to those described in section II. Figure 4, model $S$ illustrates the case where models have different shapes. Similarly, two curves could have very similar overall shape, but differ in their wiggliness. For instance, one function could be equal to another plus a high-frequency oscillation term. Figure 4, model GI illustrates this.

We will discuss the trade-offs between different models and guidelines about when each of these models is appropriate in section $\mathrm{V}$. The remainder of this section will focus on how to specify each of these five models using mgcv.

\section{Coding hierarchical GAMs in R}

Each of the models in Figure 4 can be coded straightforwardly in mgcv. We will use two example datasets to demonstrate how to code these models (see the supplemental code to reproduce these examples):

A. The $\mathrm{CO} 2$ dataset, available in $\mathrm{R}$ via the datasets package. This data is from an experimental study by Potvin, Lechowicz \& Tardif (1990) of $\mathrm{CO}_{2}$ uptake in grasses under varying concentrations of $\mathrm{CO}_{2}$, measuring how concentration-uptake functions varied between plants from two locations (Mississippi and Quebec) and two temperature treatments (chilled and warm). Twelve plants were used and $\mathrm{CO}_{2}$ uptake measured at $7 \mathrm{CO}_{2}$ concentrations for each plant (Fig. 5a). Here we will focus on how to use HGAMs to estimate inter-plant variation in functional responses. This data set has been modified from the default version available with $\mathrm{R}$, to recode the Plant variable as an unordered factor Plant_uo ${ }^{1}$.

1. A single common smoother for all observations; We will refer to this as model $G$, as it 


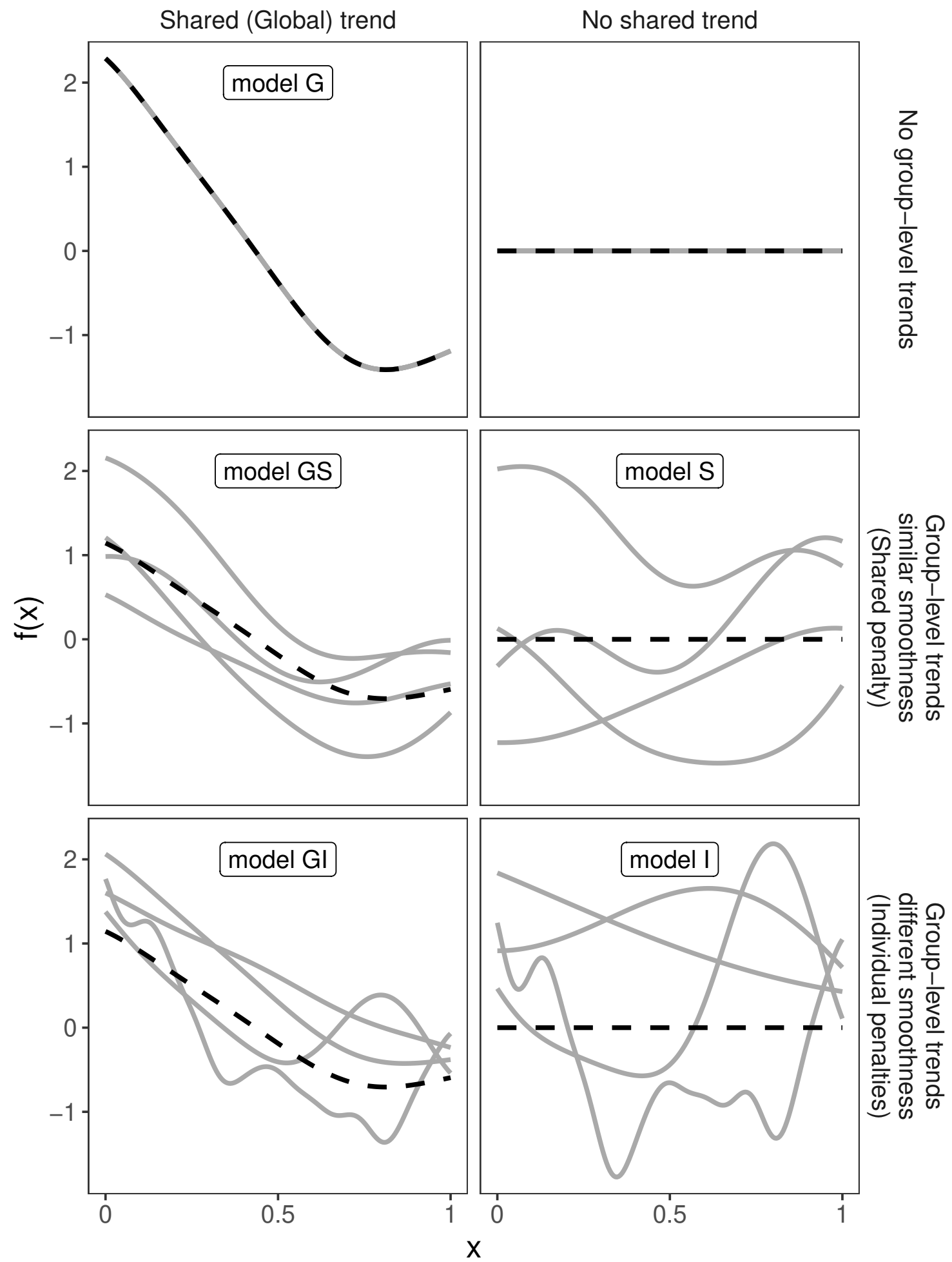

Figure 4: Alternate types of functional variation $f(x)$ that can be fitted with HGAMs. The dashed line indicates the average function value for all groups, and each solid line indicates the functional value at a given predictor value for an individual group level. The null model (of no functional relationship between the covariate and outcome, top right), is not explicitly assigned a model name. 
a

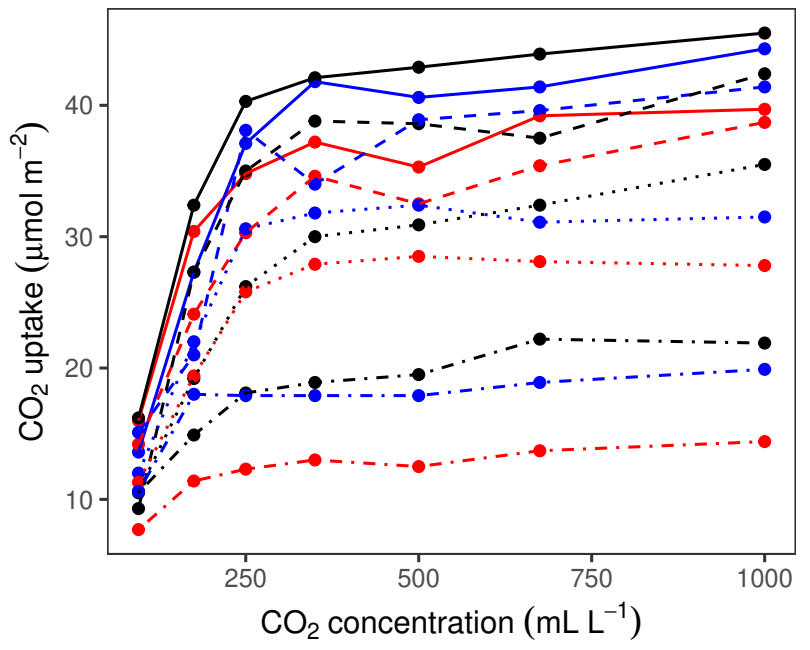

b

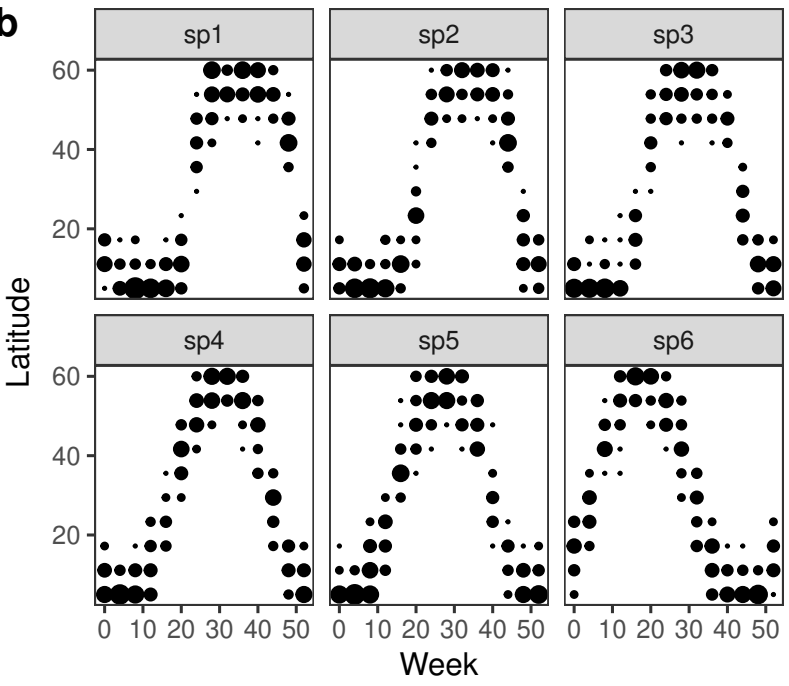

Count $\bullet 5 \bullet 10 \bullet 15$

20

Figure 5: Example data sets used throughout section III. a) Grass $\mathrm{CO}_{2}$ uptake versus $\mathrm{CO}_{2}$ concentration for 12 individual plants. Color and linetype included to distinguish individual plant trends. b) Simulated data set of bird migration, with point size corresponding to weekly counts of 6 species along a latitudinal gradient (zeros excluded for clarity).

B. Data generated from a hypothetical study of bird movement along a migration corridor, sampled throughout the year (see supplemental code). This dataset consists of simulated sample records of numbers of observed locations of 100 tagged individuals each from six species of bird, at ten locations along a latitudinal gradient, with one observation taken every four weeks. Counts were simulated randomly for each species in each location and week by creating a species-specific migration curve that gave the probability of finding an individual of a given species in a given location, then simulated the distribution of individuals across sites using a multinomial distribution, and subsampling that using a binomial distribution to simulate observation error (i.e. not every bird present at a location would be detected). The data set (bird_move) consists of the variables count, latitude, week and species (Fig. $5 b$ ). This example allows us to demonstrate how to fit these models with interactions and with non-normal (count) data. The true model used to generate this data was model GS: a single global function plus species-specific deviations around that global function.

error message if passed data coded as characters. It is also important to know whether the factor is coded as ordered or unordered (see ?factor for more details on this). This matters when fitting group-level smoothers using the by= argument (as is used for fitting models $G I$ and $I$, shown below). If the factor is unordered, mgcv will set up a model with one smoother for each grouping level. If the factor is ordered, mgcv will set any basis functions for the first grouping level to zero. In model $G I$ the ungrouped smoother will then correspond to the first grouping level, rather than the average functional response, and the group-specific smoothers will correspond to deviations from the first group. In model $I$, using an ordered factor will result in the first group not having a smoother associated with it at all. 
Throughout the examples we use Restricted Maximum Likelihood (REML) to estimate model coefficients and smoothing parameters. We strongly recommend using either REML or marginal likelihood (ML) rather than the default generalized cross-validation (GCV) criteria when fitting GAMs, for the reasons outlined in Wood (2011). In each case some data processing and manipulation has been done to obtain the graphics and results below. See supplemental code for details on data processing steps. To illustrate plots, we will be using the draw () function from the gratia package. This package was developed by one of the authors (Simpson, 2018) as a set of tools to extend plotting and analysis of mgcv models. While mgcv has plotting capabilities (through plot() methods), gratia expands these by creating ggplot2 objects (Wickham, 2016) that can be more easily extended and modified.

\section{A single common (global) smoother for all observations (Model $G$ )}

We start with the simplest model from the framework and include many details here to ensure that readers are comfortable with the terminology and $\mathrm{R}$ functions.

For our CO2 data set, we will model $\log _{e}$ (uptake) as a function of two smoothers: a TPRS of $\log _{e}$-concentration, and a random effect for Plant_uo to model plant-specific intercepts. Mathematically:

$$
\log _{e}\left(\operatorname{uptake}_{i}\right)=f\left(\log _{e}\left(\text { conc }_{i}\right)\right)+\zeta_{\text {Plant_uo }}+\varepsilon_{i}
$$

where $\zeta_{\text {Plant_uo }}$ is the random effect for plant and $\varepsilon_{i}$ is a Gaussian error term. Here we assume that $\log _{e}\left(\right.$ uptake $\left._{i}\right)$ is normally distributed.

In $\mathrm{R}$ we can write our model as:

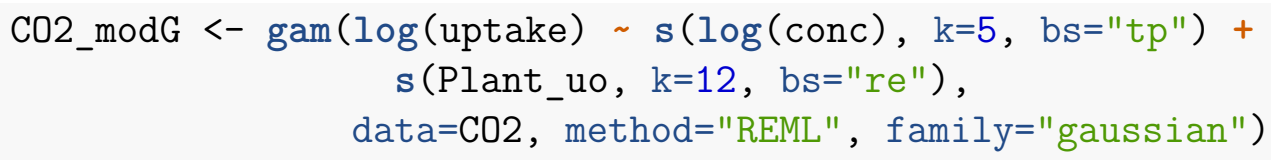

This is a common GAM structure, with a single smooth term for each variable. Specifying the model is similar to specifying a GLM in $\mathrm{R}$ via $\operatorname{glm}()$, with the addition of $\mathrm{s}($ ) terms to include one-dimensional or isotropic multidimensional smoothers. The first argument to s() are the terms to be smoothed, the type of smoother to be used for the term is specified by the bs argument, and the maximum number of basis functions is specified by $\mathrm{k}$. There are different defaults in $\mathbf{m g c v}$ for $K$, depending on the type of smoother chosen; here we use a TPRS smoother (bs="tp") for the concentration smoother, and set $k=5$ as there are only 7 separate values of concentration measured, so the default $\mathrm{k}=10$ (for TPRS) would be too high; further, setting $\mathrm{k}=5$ saves on computational time (see section $\mathrm{V}$ ). The random effect smoother (bs="re") that we used for the Plant_uo factor always has a $\mathrm{k}$ value equal to the number of levels in the grouping variable (here, 12). We specified $k=12$ just to make this connection apparent.

Figure 6 illustrates the output of gratia's draw() function for C02_modG: the panel labelled $\mathrm{s}(\log (\mathrm{conc}))$ shows the estimated smoother for concentration, and the panel labelled 

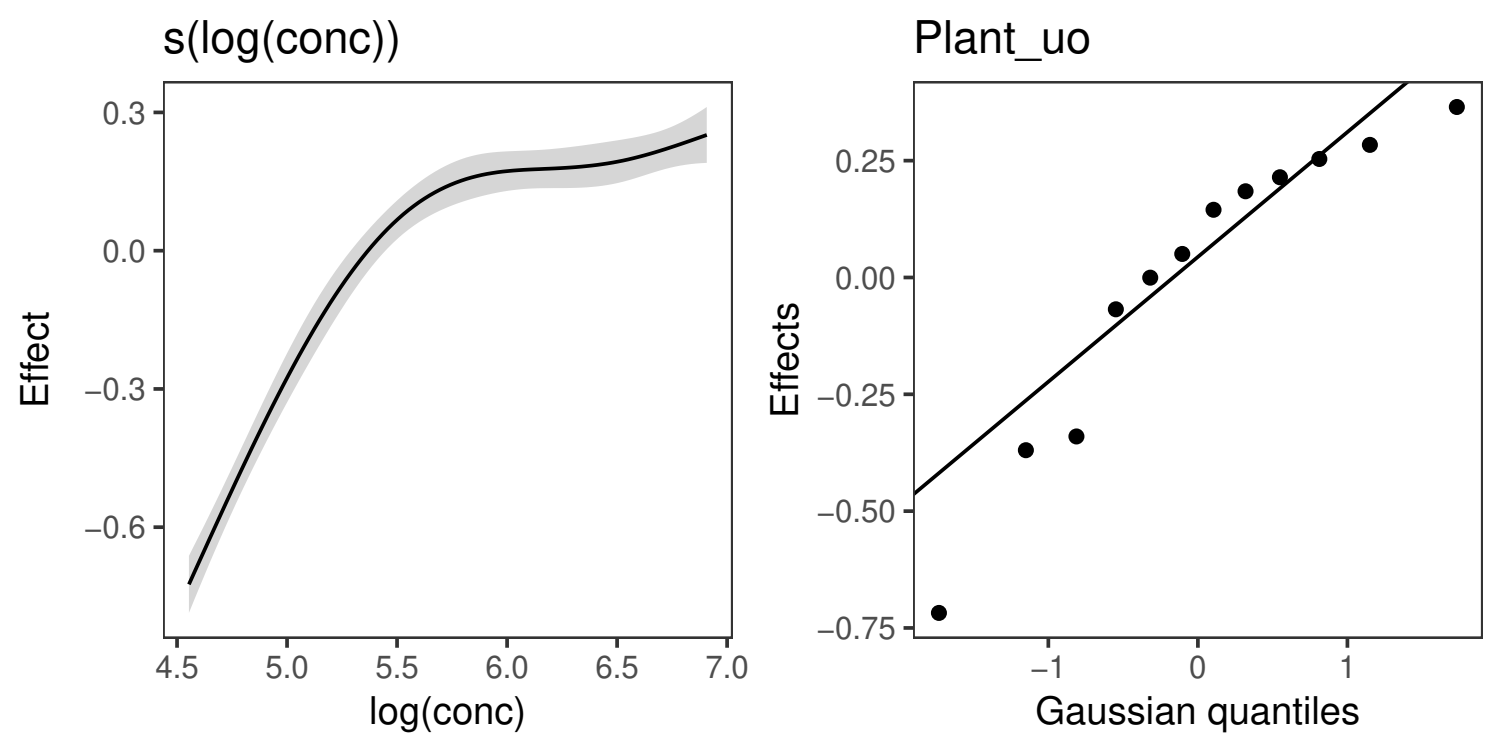

Figure 6: gratia plotting output for model $G$ applied to the CO2 dataset. s $(\log (\operatorname{conc}))$ : the smoother of $\log _{e}$ concentration. Plant_uo: a quantile-quantile plot of the random effects against Gaussian quantiles, used to check the appropriateness of the normal random effect assumption.

\footnotetext{
${ }^{2}$ The cyclic smoother requires that the start and end points of the cyclic variable are specified, via the
}

Plant_uo shows a quantile-quantile plot of the estimated random effects vs. Gaussian quantiles, which can be used to check our model.

Looking at the effects by term is useful, but we are often interested in fitted values or predictions from our models. Using the built in prediction functions with $\mathbf{m g c v}$, we can estimate what the fitted function (and uncertainty around it) should look like for each level, as shown in Figure 7 (see supplemental code for more details on how to generate these predictions).

Examining these plots, we see that while functional responses among plants are similar, some patterns are not captured by this model. For instance, for plant Qc2 the model clearly underestimates $\mathrm{CO} 2$ uptake. A model including individual differences in functional responses may better explain variation.

For our bird example, we model the count of birds as a function of location and time, including their interaction. For this we structure the model as:

$$
\mathbb{E}\left(\text { count }_{i}\right)=\exp \left(f\left(\text { week }_{i}, \text { latitude }_{i}\right)\right)
$$

where we assume that count $_{i} \sim$ Poisson. For the smooth term, $f$, we employ a tensor product of latitude and week, using a TPRS for the marginal latitude effect, and a cyclic CRS for the marginal week effect to account for the cyclic nature of weekly effects (we expect week 1 and week 52 to have very similar values) $)^{2}$, both splines had basis complexity (k) of 10 . 

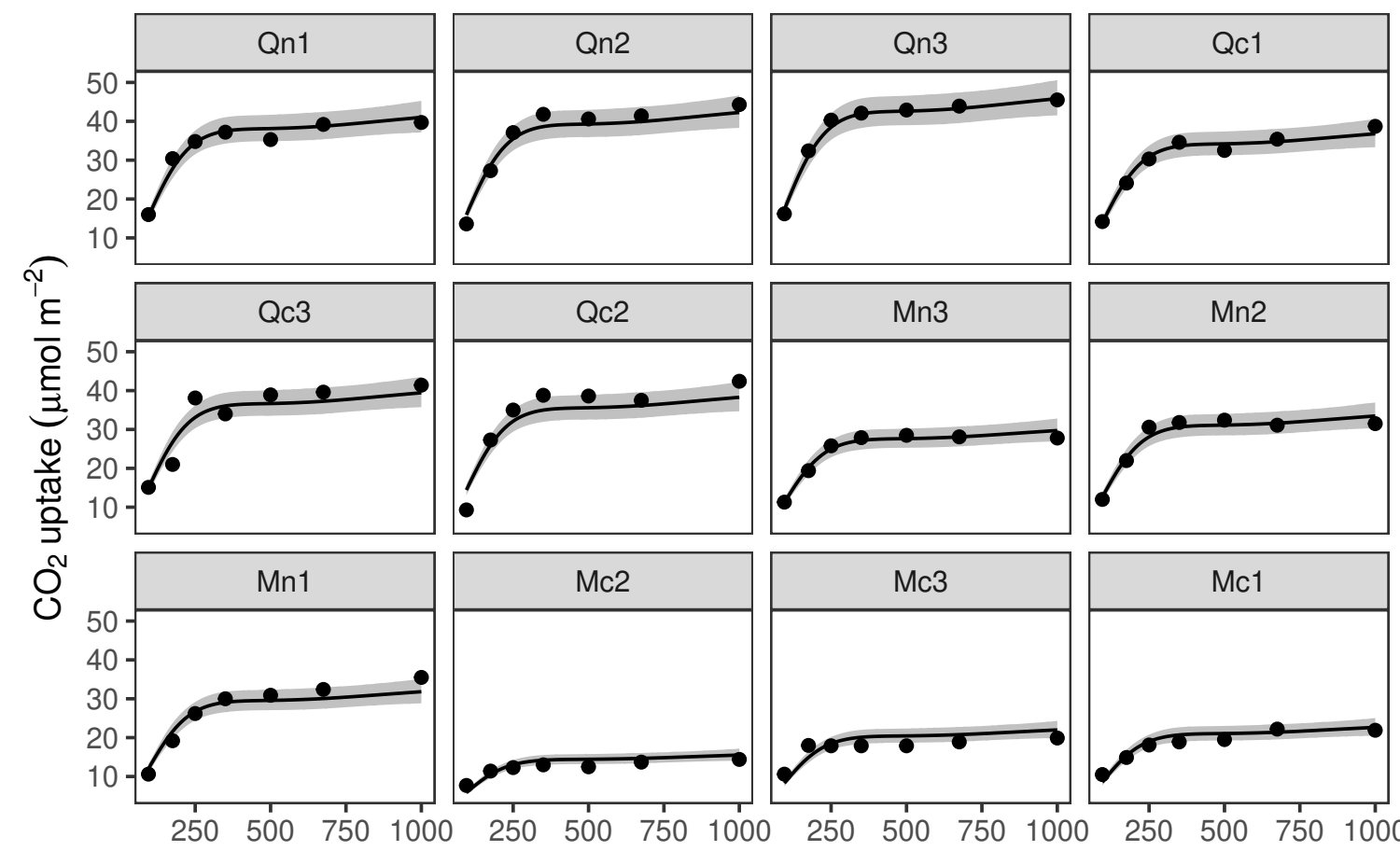

$\mathrm{CO}_{2}$ concentration $\left(\mathrm{mL} \mathrm{L}^{-1}\right)$

Figure 7: Predicted uptake function ( \pm 2 s.e.) for each plant, based on model $G$ (a single global function for uptake plus a individual-level random effect intercept). Model predictions are for log-uptake, but are transformed here to show the fitted function on the original scale of the data. 


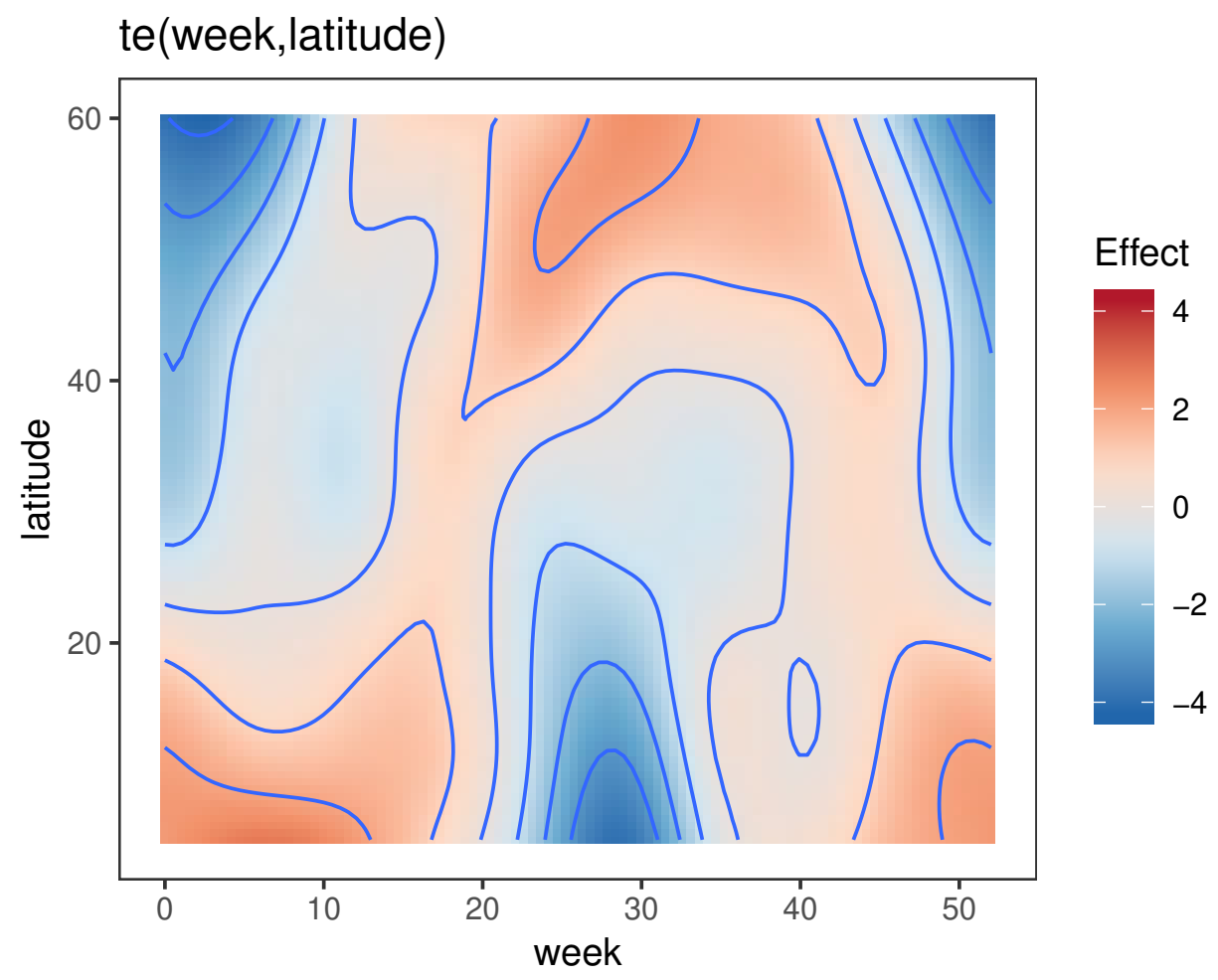

Figure 8: Plot illustrating the average log-abundance of all bird species at each latitude for each week, with red colours indicating more individuals and blue colours fewer.

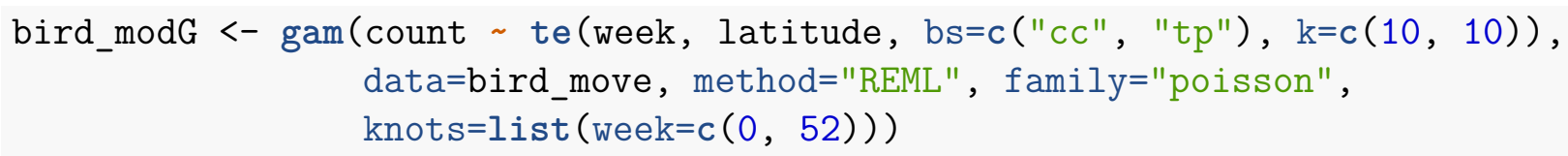

knots argument; the smoother will have the exact same value at the start and end. In the absence of a specified start and end point, gam will assume the end points are the smallest and largest observed levels of the covariate (see mgcv: :smooth.construct.cc.smooth.spec for more details). Note that in bird_modG we have specified week 0 and week 52 as the endpoints, as the first (week 1) and last weeks (week 52) of the year should not have exactly the same expected value. 

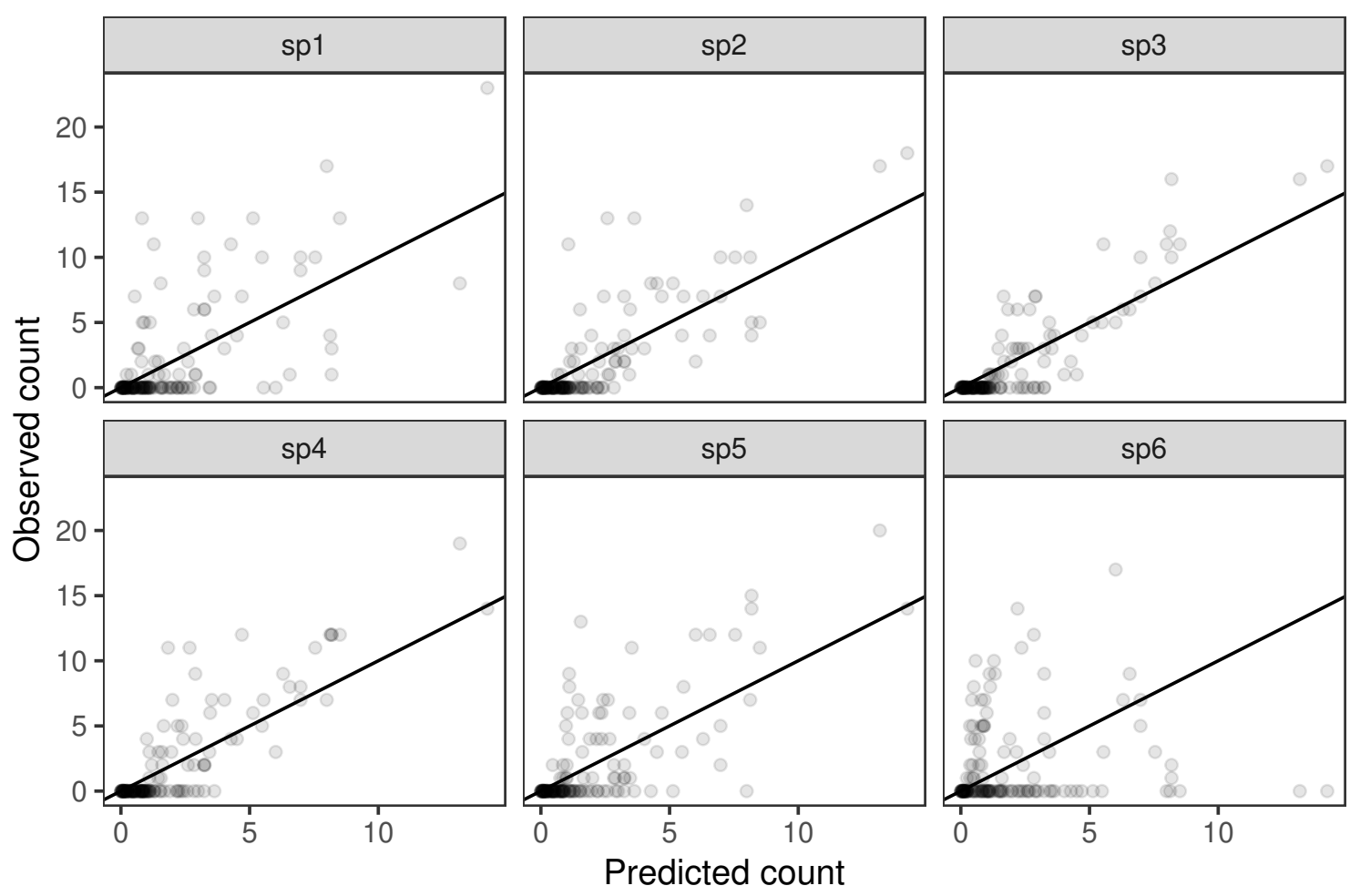

Figure 9: Observed counts by species versus predicted counts from bird_modG (1-1 line added as reference). If our model fitted well we would expect that all species should show similiar patterns of dispersion around the 1-1 line (and as we are assuming the data is Poisson, the variance around the mean should equal the mean). Instead we see that variance around the predicted value is much higher for species 1 and 6 .

timing. The rest of this section will focus on how to model this type of variation.

\section{A single common smoother plus group-level smoothers that have the same wig- gliness (model $G S$ )}

Model $G S$ is a close analogue to a GLMM with varying slopes: all groups have similar functional responses, but inter-group variation in responses is allowed. This approach works by allowing each grouping level to have its own functional response, but penalizing functions that are too far from the average.

This can be coded in mgcv by explicitly specifying one term for the global smoother (as in model $G$ above) then adding a second smooth term specifying the group-level smooth terms, using a penalty term that tends to draw these group-level smoothers towards zero. mgcv provides an explicit basis type to do this, the factor-smoother interaction or " $f \mathrm{~s}$ " basis (see ?mgcv: :factor.smooth.interaction for details). This smoother creates a copy of each set of basis functions for each level of the grouping variable, but only estimates one smoothing parameter for all groups. To ensure that all parts of the smoother can be shrunk towards 

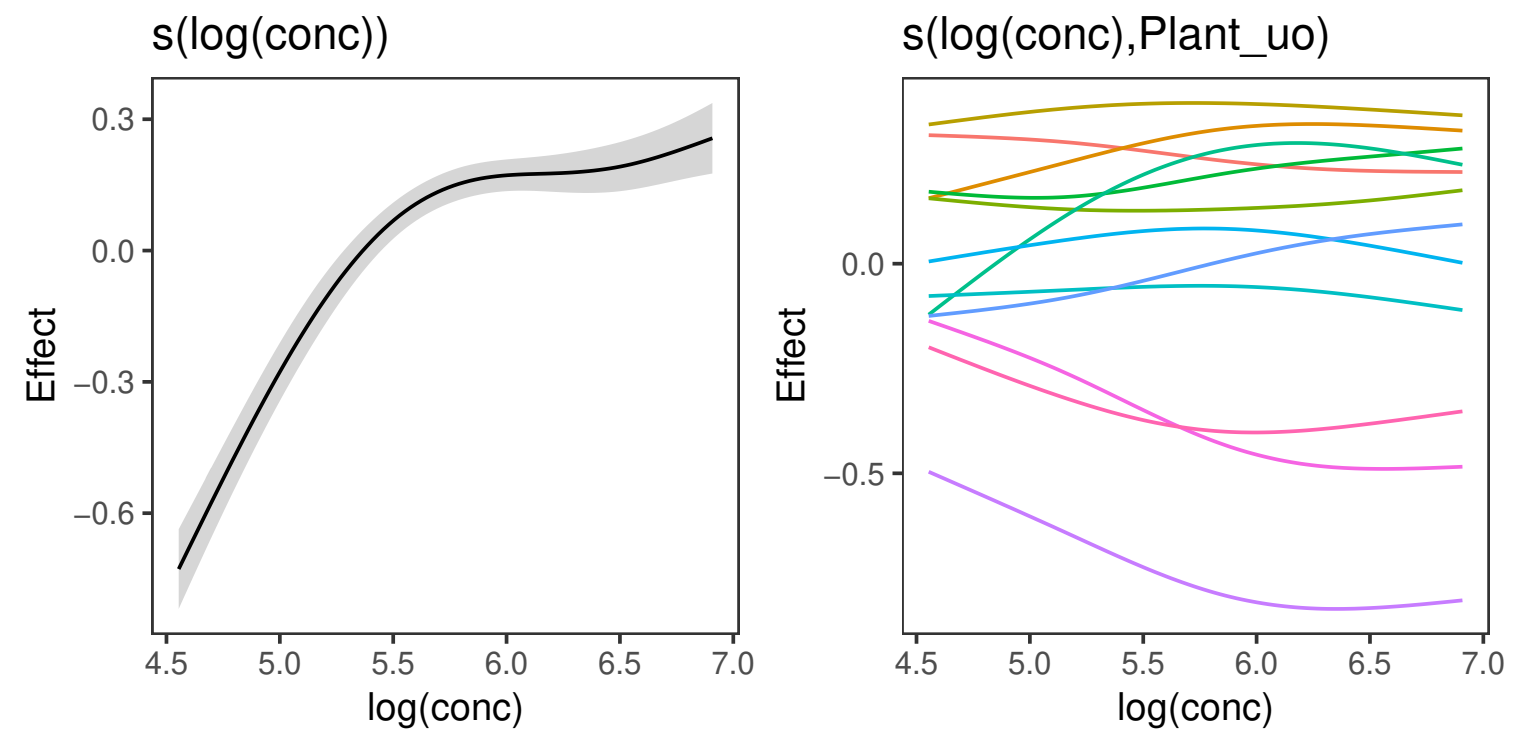

Figure 10: Global function $(\mathrm{s}(\log (\operatorname{con} \mathrm{c})))$ and group-specific deviations from the global function $(\mathrm{s}(\log (\mathrm{conc}), \mathrm{Plant}$ uo $))$ for CO2_modGS

\footnotetext{
${ }^{3}$ As part of the penalty construction, each group will also have its own intercept (part of the penalized null space), so there is no need to add a separate term for group specific intercepts as we did in model $G$.

${ }^{4}$ As mentioned in section II, these terms can be specified either with te() or t2() terms. Using t2 as
}

where $f_{\mathrm{Plant}_{-} \mathrm{uo}_{i}}\left(\log _{e}\left(\right.\right.$ conc $\left.\left._{i}\right)\right)$ is the smoother for concentration for the given plant. In $\mathrm{R}$ we then have:

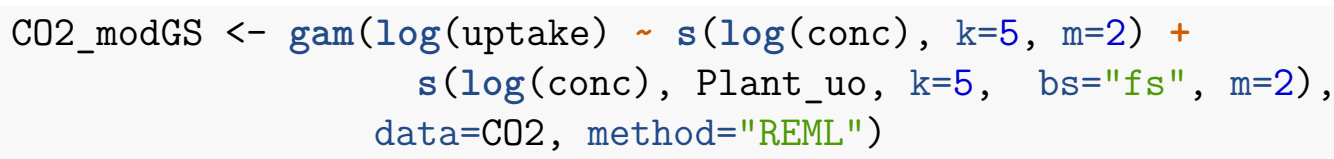

Figure 10 shows the fitted smoothers for C02_modGS. The plots of group-specific smoothers indicate that plants differ not only in average log-uptake (which would correspond to each plant having a straight line at different levels for the group-level smoother), but differ slightly in the shape of their functional responses. Figure 11 shows how the global and group-specific smoothers combine to predict uptake rates for individual plants. We see that, unlike in the single global smoother case above, none of the curves deviate from the data systematically.

The factor-smoother interaction-based approach mentioned above does not work for higherdimensional tensor product smoothers ( $f_{s}()$ does still work for higher dimensional isotropic smoothers). Instead, the group-specific term can be specified with a tensor product of the continuous smoothers and a random effect for the grouping parameter ${ }^{4}$. e.g.: 


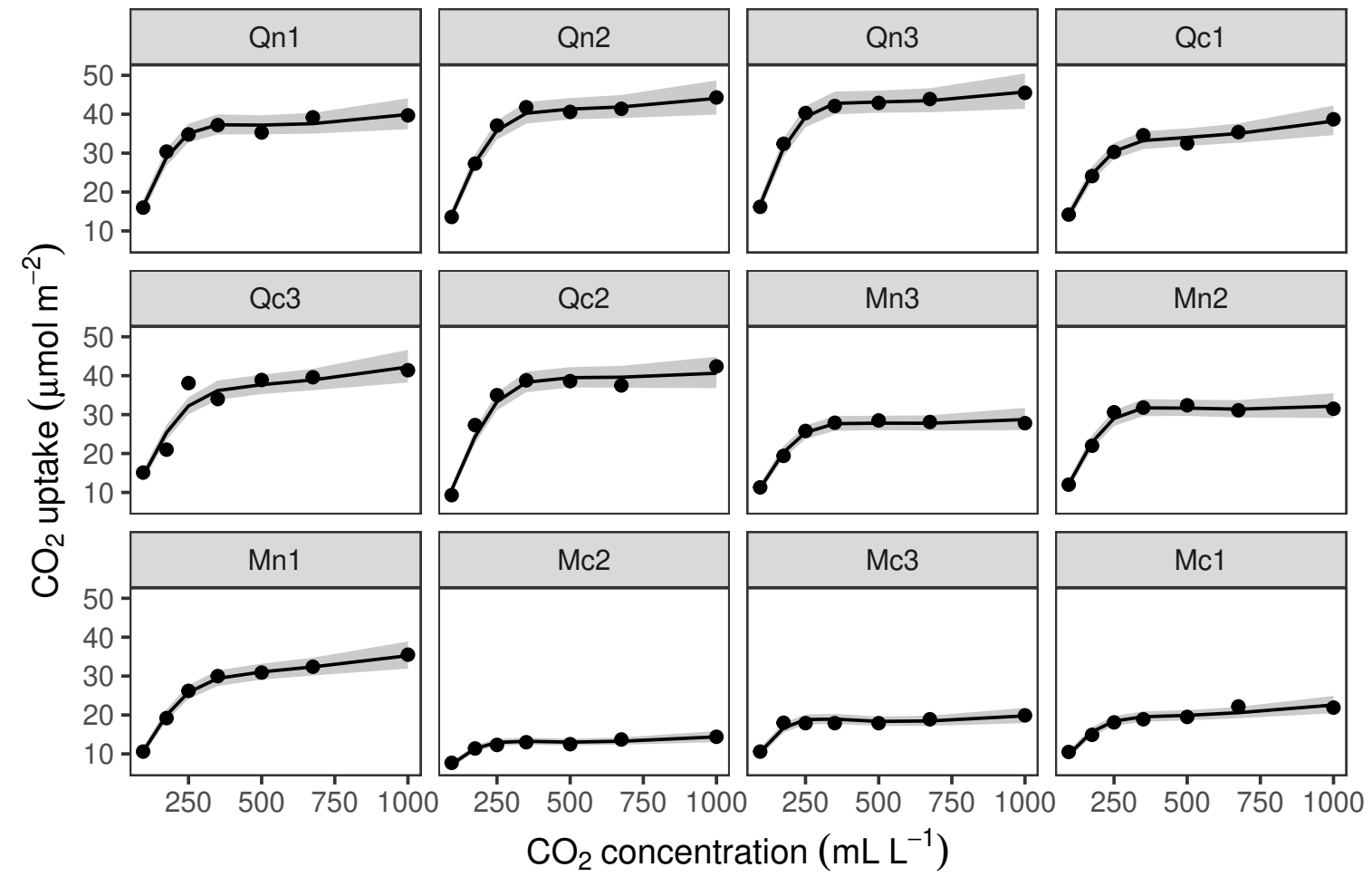

Figure 11: Predicted uptake values (lines) versus observed uptake for each plant, based on model $G S$. 

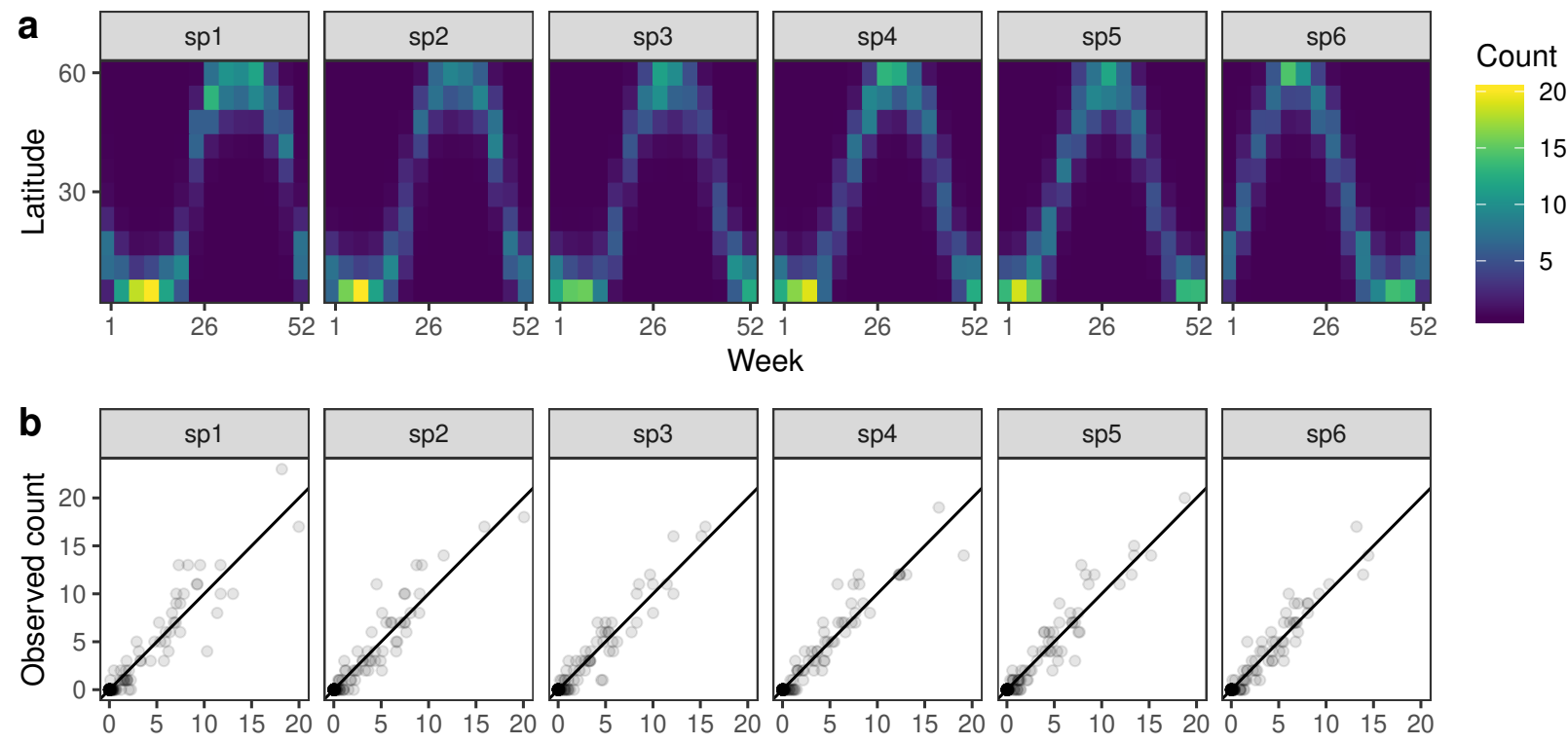

Predicted count (model ${ }^{*} \mathrm{GS}{ }^{*}$ )

Figure 12: a) Predicted migration paths for each species based on bird_modGS, with lighter colors corresponding to higher predicted counts. b) Observed counts versus predictions from bird_modGS.

$y \sim t e(x 1, x 2, b s=" t p ", m=2)+$ t2(x1, x2, fac, bs=c("tp", "tp", "re"), m=2, full=TRUE)

We illustrate this approach below on the bird migration data.

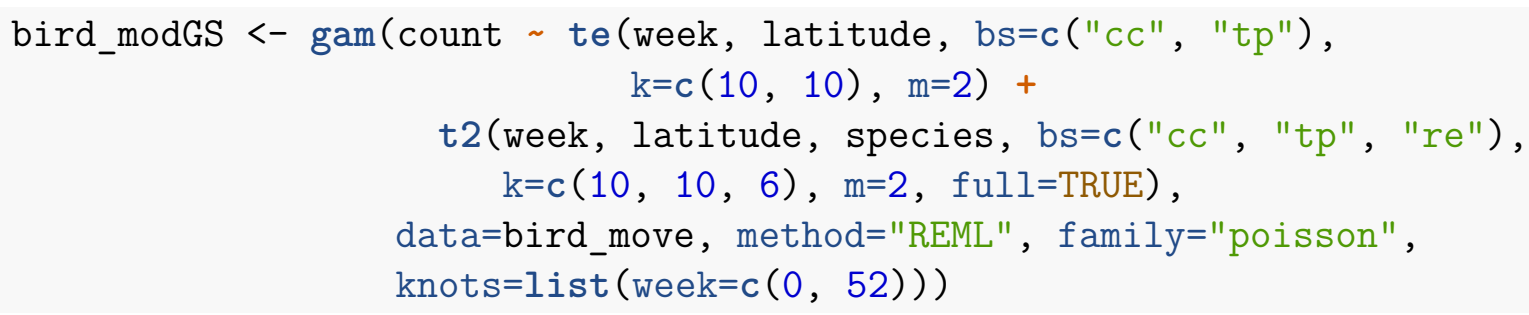

Model $G S$ is able to effectively capture the observed patterns of interspecific variation in migration behaviour (Fig. 12a). It shows a much tighter fit between observed and predicted values, as well as less evidence of over-dispersion in some species compared to model $G$ (Fig. $12 \mathrm{~b})$.

above (with full=TRUE) is essentially a multivariate equivalent of the factor-smoother interaction; it requires more smooth terms than te(), but can be fit using other mixed effects software such as lme4, which is useful when fitting models with a large number of group levels (see Section V on computational issues for details). We have generally found that $\mathrm{t} 2$ (full=TRUE) is the best approach for multidimensional GS models when the goal is to accurately estimate the global smoother in the presence of group-level smoothers; other approaches (using te ()$)$ tend to result in the global smoother being overly penalized toward the flat function, and the bulk of the variance being assigned to the group-level smoother. We discuss this further in section V, "Estimation issues when fitting both global and group-level smoothers". 


\section{A single common smoother plus group-level smoothers with differing wiggliness (Model GI)}

This model class is very similar to model $G S$, but we now allow each group-specific smoother to have its own smoothing parameter and hence its own level of wiggliness. This increases the computational cost of the model (as there are more smoothing parameters to estimate), and means that the only information shared between groups is through the global smoother, the common error term, and through the random effect for group-level intercepts (if used). This is useful if different groups differ substantially in how wiggly they are.

Fitting a separate smoother (with its own penalties) can be done in mgcv by using the by argument in the $\mathbf{s}()$ and te() (and related) functions. Therefore, we can code the formula for this model as:

$y \sim s(x, b s=" t p ")+s(x, b y=f a c, m=1, b s=" t p ")+s(f a c, b s=" r e ")$

Note two major differences here from how model $G S$ was specified:

1. We explicitly include a random effect for the intercept (the $b s=" r e "$ term), as groupspecific intercepts are not incorporated into factor by variable smoothers (as would be the case with a factor smoother or a tensor product random effect).

2. We specify $m=1$ instead of $m=2$ for the group-level smoothers, which means the marginal TPRS basis for this term will penalize the squared 1st derivative of the function, rather than the second derivative. This, also, reduces co-linearity between the global smoother and the group-specific terms which occasionally leads to high uncertainty around the global smoother (see section $\mathrm{V}$ for more details). TPRS with $\mathrm{m}=1$ have a more restricted null space than $\mathrm{m}=2$ smoothers, so should not be as collinear with the global smoother (Wieling et al., 2016; Baayen et al., 2018). We have observed that this is much more of an issue when fitting model GI compared to model GS.

We modify the CO2 model to follow this approach like so:

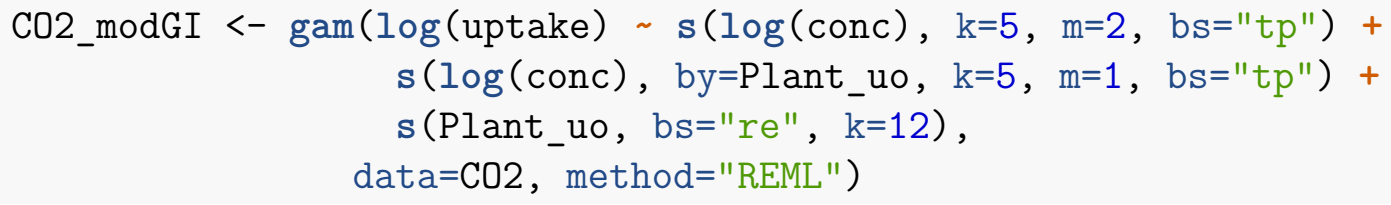

Figure 13 shows a subsample of the group-specific smoothers from this model. It is apparent from this that some groups (e.g. Qc1) have very similar shapes to the global smoother (differing only in intercept), others do differ from the global trend, with higher uptake at low concentrations and lower uptake at higher concentrations (e.g. Mc1, Qn1), or the reverse pattern (e.g. Mn1).

Using model GI with higher-dimensional data is also straightforward; by terms work just as well in tensor-product smoothers as they do with isotropic smoothers. We can see this with our bird model:

bird_modGI <- gam(count species +

te(week, latitude, $b s=c(" c c ", " t p "), k=c(10,10), m=2)+$ 

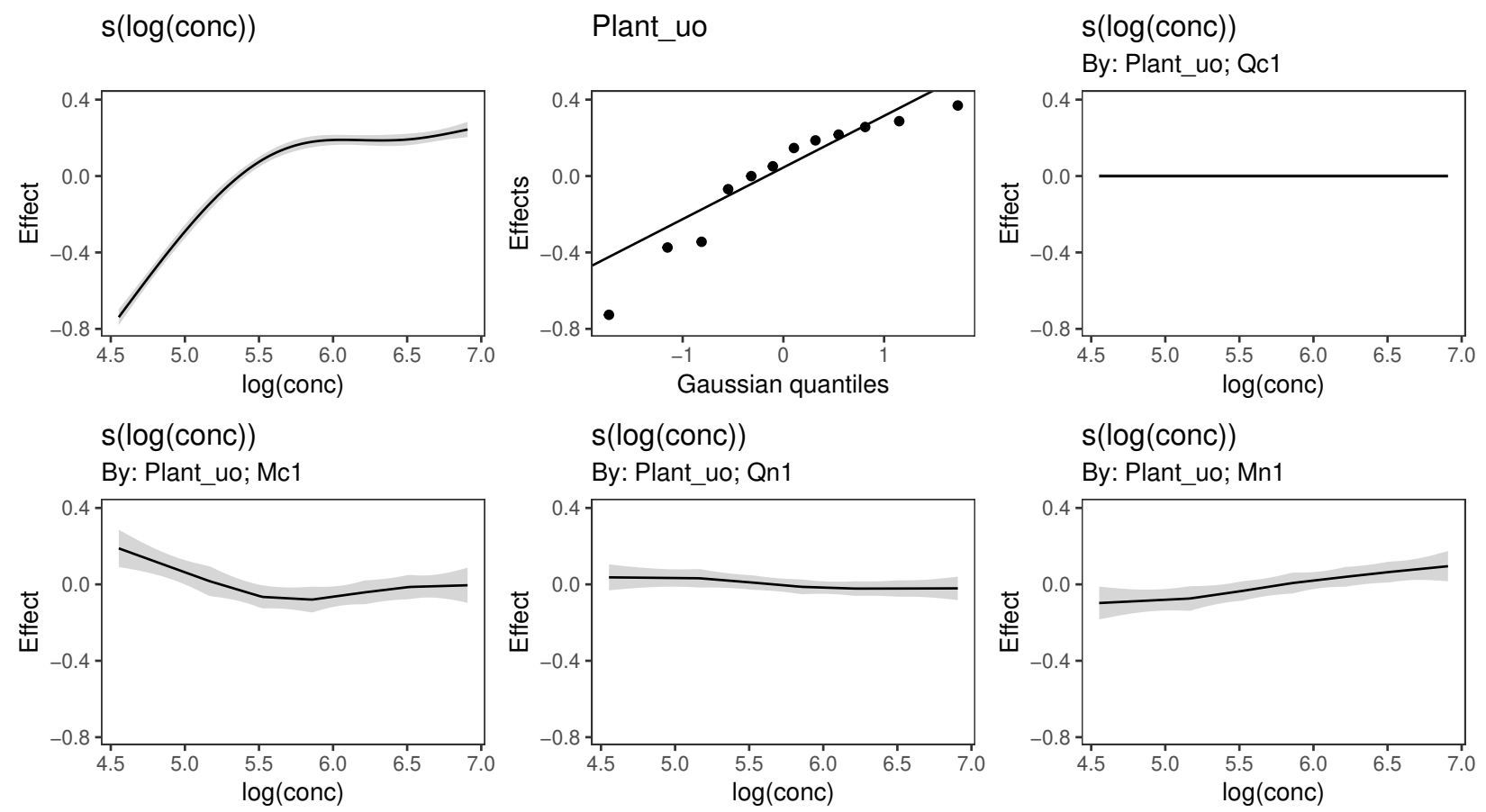

Figure 13: Functional relationships for the CO2 data estimated for model GI. s( $\log (\operatorname{conc}))$ : the global smoother; Plant_uo: species-specific random effect intercepts. The remaining plots are a selected subset of the plant-specific smoothers, indicating how the functional response of that plant differs from the global smoother. 


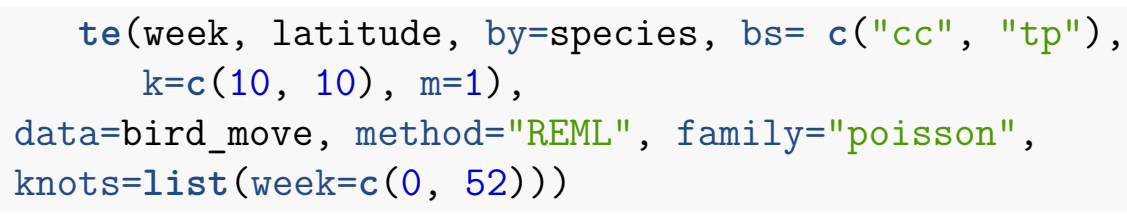

As above, we have set $(\mathrm{m}=1)$ for the latitude marginal effect to avoid issues of collinearity between the global and group-level smoother. Note that switching $\mathrm{m}=1$ to $\mathrm{m}=2$ does not have any effect on the marginal basis for week, where we are using a cyclic smoother instead of a TPRS.

The fitted model for bird_modGI is visually indistinguishable from bird_modGS (Fig. 12) so we do not illustrate it here.

\section{Models without global smoothers (models $S$ and $I$ )}

We can modify the above models to exclude the global term (which is generally faster; see section $\mathrm{V}$ ). When we do not model the global term, we are allowing each group to be differently shaped without restriction. Though there may be some similarities in the shape of the functions, these models' underlying assumption is that group-level smooth terms do not share or deviate from a common form.

\section{Model $S$ :}

Model $S$ (shared smoothers) is model $G S$ without the global smoother term; this type of model takes the form: $y \sim s(x, f a c, b s=" f s ")$ or $y \sim t e(x 1, x 2, f a c, b s=c(" t p ", ~ " t p "$, "re") in mgcv. This model assumes all groups have the same smoothness, but that the individual shapes of the smooth terms are not related. Here we do not plot these models; the model plots are very similar to the plots for model $G S$. This will not always be the case. If in a study there are very few data points in each grouping level (relative to the strength of the functional relationship of interest), estimates from model $S$ will typically be much more variable than from model $G S$; there is no way for the model to share information on function shape between grouping levels without the global smoother. See section V on computational issues for more on how to choose between different models.

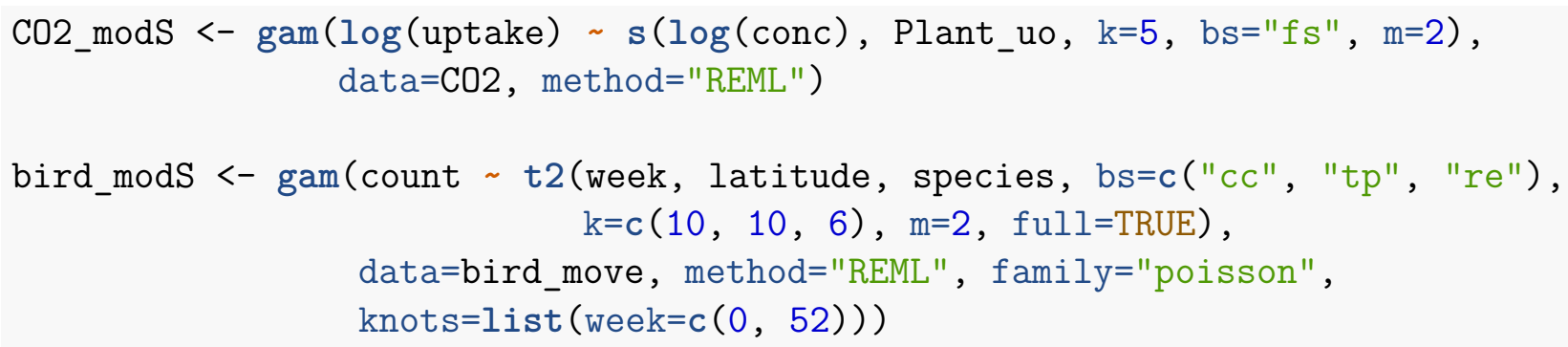

\section{Model I:}

Model $I$ is model $G I$ without the first term: $\mathrm{y} \sim \mathrm{fac}+\mathrm{s}(\mathrm{x}, \mathrm{by}=\mathrm{fac})$ or $\mathrm{y} \sim \mathrm{fac}+\mathrm{te}(\mathrm{x} 1, \mathrm{x} 2$, by=fac) (as above, plots are very similar to model GI). 


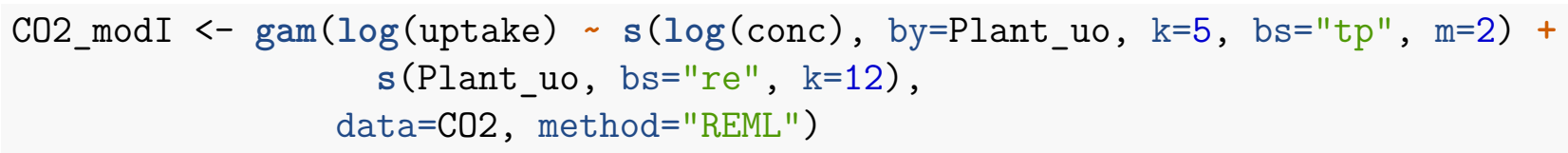

bird_modI <- gam(count species + te(week, latitude, by=species, $\mathrm{bs}=c(" c c ", " t p "), \mathrm{k}=\mathrm{c}(10,10), \mathrm{m}=2)$, data=bird_move, method="REML", family="poisson", knots $=$ list $($ week $=c(0,52))$ )

\section{Comparing different HGAM specifications}

These models can be compared using standard model comparison tools. Model $G S$ and model $G I$ will generally be nested in model $G$ (depending on how each model is specified) so comparisons using generalized likelihood ratio tests (GLRTs) may be used to test if group-level smoothers are necessary (if fit using method="ML"). However, we do not currently recommend this method. There is not sufficient theory on how accurate parametric p-values are for comparing these models; there is uncertainty about what degrees of freedom to assign to models with varying smoothness, and slightly different model specifications may not result in nested models. (See Wood (2017a) Section 6.12.4 and ?mgcv: : anova.gam for more discussion on using GLRTs to compare GAMs.)

Comparing models based on AIC is a more robust approach to comparing the different model structures. There is well-developed theory of how to include effects of penalization and smoothing parameter uncertainty when estimating the model complexity penalty for AIC (Wood, Pya \& Säfken, 2016). We demonstrate this approach in Table 1. Using AIC, there is strong support for including among-group functional variability for both the CO2 dataset and the bird_move dataset (compare models $G$ versus all other models). For the CO2 dataset (Table 1A), there is relatively strong evidence that there is more inter-group variability in smoothness than model $G S$ allows, and weaker evidence that model $S$ or $I$ (separate smoothers for all plants) show the best fit.

For the bird_move dataset (Table 1B), model GS (global smoother plus group-level smoothers with a shared penalty) gives the best fit for all models including a global smooth (which is good as we simulated the data from a model with this structure!). However, model $S$ (without a global term) still fits this data better than model $G S$ based on AIC. This highlights an issue with AIC for selecting between models with and without a global smooth: as it is possible to fully recreate the global term by just allowing each group-level smoother to have a similar shape to one another (that is, the global term is totally concurve with the group-level smoothers; see section V) model selection criteria such as AIC may indicate that the extra parameters required to fit the global smoother are unnecessary ${ }^{5}$.

\footnotetext{
${ }^{5}$ If it is important for a given study to determine if there is evidence for a significant global smooth effect, we recommend fitting model $G S$ or $G I$, including the argument select = TRUE in the gam function. This has the effect of adding an extra penalty to each smooth term, that penalizes functions in the null space of the penalty matrices for each smooth. By doing this, it is possible for mgcv to penalize all model terms to a
} 
Table 1: AIC table comparing model fits for example datasets

\begin{tabular}{lrrr}
\hline Model & df & AIC & deltaAIC \\
\hline A. CO2 models & & & \\
CO2_modG & 17 & -119 & 101 \\
CO2_modGS & 39 & -199 & 22 \\
CO2_modGI & 42 & -216 & 4 \\
CO2_modS & 53 & -219 & 1 \\
CO2_modI & 56 & -220 & 0 \\
B. bird_move & models & \\
bird_modG & 51 & 3374 & 1823 \\
bird_modGS & 140 & 1554 & 4 \\
bird_modGI & 208 & 1682 & 132 \\
bird_modS & 127 & 1550 & 0 \\
bird_modI & 200 & 1634 & 84 \\
\hline
\end{tabular}

Given this issue with selecting global terms, we strongly recommend not selecting models based purely on AIC. Instead, model selection should be based on expert subject knowledge about the system, computational time, and most importantly, the inferential goals of the study. Table 1A indicates that models $S$ and $I$ (which do not have a global function) fit the C02 data better than models with a global function, and that model $S$ fits the bird_move data better than model GS. However, it is the shape of the global function that we are actually interested in here, as models $S$ and $I$ cannot be used to predict the concentration-uptake relationship for plants that are not part of the training set, or the average migration path for birds. The same consideration holds when choosing between model $G S$ and GI: while model GI fits the C02 data better than model GS (as measured by AIC), model GS can be used to simulate functional variation for unobserved group levels, whereas this is not possible within the framework of model GI. The next section works through two examples to show how to choose between different models, and section $\mathrm{V}$ discusses these and other model fitting issues in more depth.

It also is important to recognize that AIC, like any function of the data, is a random variable and should be expected to have some sampling error (Forster \& Sober, 2011). In cases when the goal is to select the model that has the best predictive ability, we recommend holding some fraction of the data out prior to the analysis and comparing how well different models fit that data, or using $k$-fold cross validation as a more accurate guide to how well a given model may predict out of sample. Predictive accuracy may also be substantially improved by averaging over multiple models (Dormann et al., 2018).

zero effect, in effect doing variable selection (Marra \& Wood, 2011). When select=TRUE, the significance of the global term can be found by looking at the significance of the term in summary.gam (model). Note that this can significantly increase the amount of time it takes to fit a model for data sets with a large number of penalty terms (such as model GI when the number of groups is high). 


\section{${ }^{481}$ IV: Examples}

We now demonstrate two worked examples on one data set to highlight how to use HGAMs in practice, and to illustrate how to fit, test, and visualize each model. We will demonstrate how to use these models to fit community data, to show when using a global trend may or may not be justified, and to illustrate how to use these models to fit seasonal time series.

For these examples, data are from a long-term study in seasonal dynamics of zooplankton, collected by the Richard Lathrop. The data were collected from a chain of lakes in Wisconsin (Mendota, Monona, Kegnonsa, and Waubesa) approximately bi-weekly from 1976 to 1994. They consist of samples of the zooplankton communities, taken from the deepest point of each lake via vertical tow. The data are provided by the Wisconsin Department of Natural Resources and their collection and processing are fully described in Lathrop (2000).

Zooplankton in temperate lakes often undergo seasonal cycles, where the abundance of each species fluctuates up and down across the course of the year, with each species typically showing a distinct pattern of seasonal cycles. The inferential aims of these examples are to $(i)$ estimate variability in seasonality among species in the community in a single lake (Mendota), and (ii) estimate among-lake variability for the most abundant taxon in the sample (Daphnia mendotae) across the four lakes. To enable evaluation of out-of-sample performance, we split the data into testing and training sets. As there are multiple years of data, we used data from the even years to fit (train) models, and the odd years to test the fit.

Each record consists of counts of a given zooplankton taxon taken from a subsample from a single vertical net tow, which was then scaled to account for the relative volume of subsample versus the whole net sample and the area of the net tow, giving population density per $m^{2}$. Values are rounded to the nearest 1000. Observed densities span four orders of magnitude. We modelled density using a Gamma distribution with a log-link. For any net tow sample where a given taxon was not observed, we set that taxon's density to 1000 (the minimum possible sample size $)^{6}$. To evaluate how well each model fits new data (not used to fit the model), we calculated the total deviance of the out-of-sample data that we had previously held out. The deviance is equal to two times the sum of the difference between the log-likelihood of the out-of-sample data (as predicted by each model) and a saturated model, that has one predictor for each data point, all multiplied by the scale parameter for the family of interest. It can be interpreted similarly to the residual sum of squares for a simple linear regression (Wood, 2017a, p. 109).

First, we demonstrate how to model community-level variability in seasonality, by regressing scaled density on day of year with species-specific curves. As we are not interested in average seasonal dynamics, we focus on models $S$ and $I$ (if we wanted to estimate the seasonal dynamics for rarer species, adding a global smooth term might be useful, so we could borrow information from the more common species). As the data are seasonal, we use cyclic smoothers as the

\footnotetext{
${ }^{6} \mathrm{~A}$ more appropriate model for this data would be to assume that density is left censored, where 1000 is treated as a threshold which the data may lie below, but it is not possible to measure lower than this. However, mgcv does not currently have a left-censored family. The brms package, for Bayesian model fitting, can fit a left-censored Gamma distribution, so it would be possible to fit this model using that software. We discuss using HGAMs in brms in section V.
} 
basis for seasonal dynamics. Therefore we need to specify start and end points for our cycles using the knots argument to gam(), as well as specify this smoother type as a factor-smooth interaction term using the xt argument (the xt argument is how any extra information that a smoother might need is supplied; see ?mgcv: :s for more information). Note that we also include a random effect smoother for both taxon and taxon:year_f, where year_f is year transformed into a factor variable. This deals with the fact that average zooplankton densities can show large year-to-year variation. The argument drop. unused. levels=FALSE is also included so the gam function does not drop the year factor levels corresponding to those in the held-out test data set.

\section{Model $S$ :}

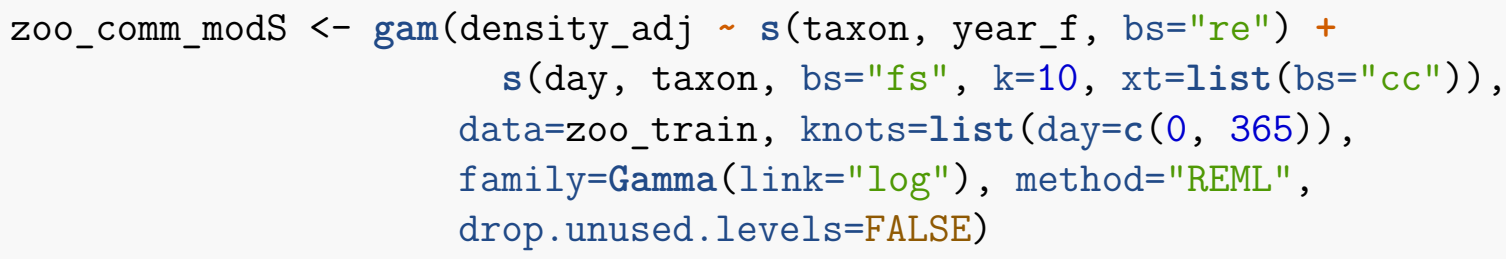

\section{Model I:}

\# Note that $s$ (taxon, bs="re") has to be explicitly included here, as the \# day by taxon smoother does not include an intercept zoo_comm_modI <- gam(density_adj s(day, by=taxon, k=10, bs="cc") + $s($ taxon, bs="re") + s(taxon, year_f, bs="re"), data=zoo_train, knots=list $($ day $=c(0,365))$, family=Gamma (link="log"), method="REML", drop. unused . levels=FALSE)

At this stage of the analysis (prior to model comparisons), it is useful to determine if any of the fitted models adequately describe patterns in the data (i.e. goodness of fit testing). mgcv's gam. check() facilitates this model-checking. This function creates a set of standard diagnostic plots: a QQ plot of the deviance residuals (see Wood (2017a)) compared to their theoretical expectation for the chosen family, a plot of response versus fitted values, a histogram of residuals, and a plot of residuals versus fitted values. It also conducts a test for each smooth term to determine if the number of degrees of freedom ( $\mathrm{k}$ ) for each smooth is adequate (see ?mgcv: :gam. check for details on how that test works). The code for checking model $S$ and $I$ for the community zooplankton model is:

gam.check(zoo_comm_modS)

gam. check (zoo_comm_modI)

We have plotted QQ plots and fitted-versus residual plots for model I (fitted versus response plots are generally less useful for non-normally distributed data as it can be difficult to visually assess if the observed data shows more heteroskedasticity than expected). The results for model $S$ are virtually indistinguishable to the naked eye. We have also used alternate 

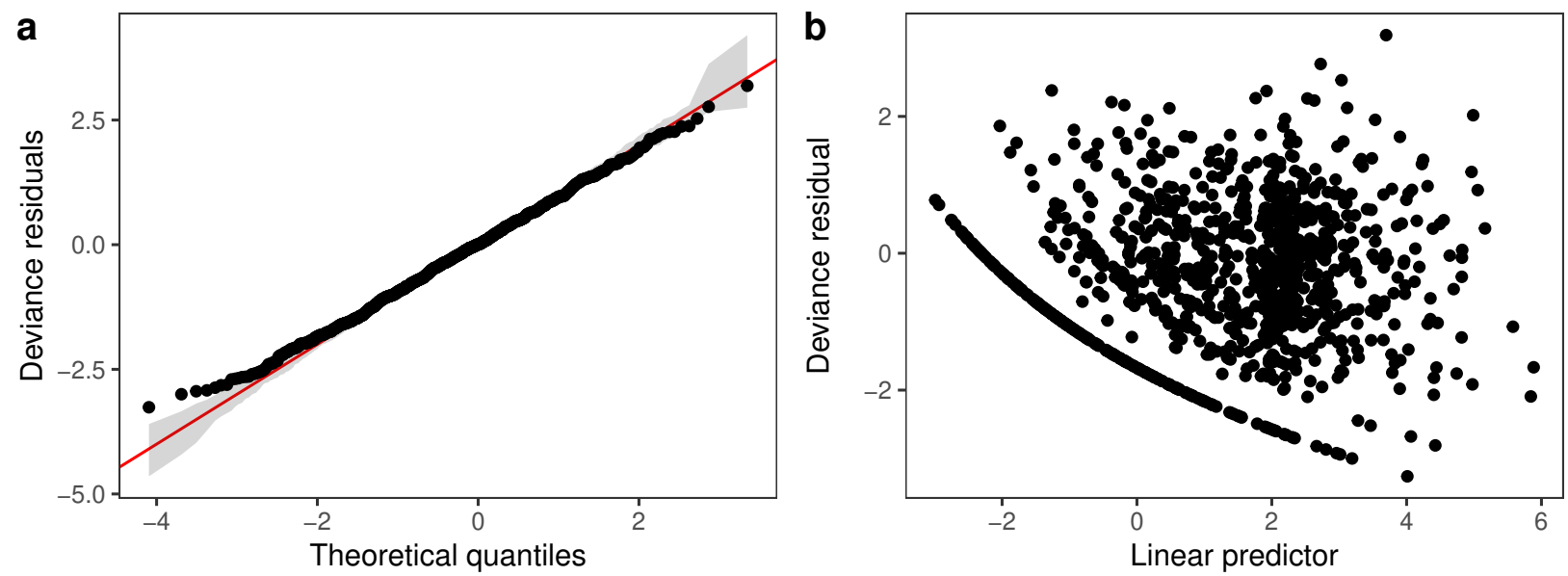

Figure 14: Diagnostic plots for model $I$ fitted to zooplankton community data in Lake Mendota. a) QQ-plot of residuals (black). Red line indicates the 1-1 line and grey bands correspond to the expected 95\% CI for the QQ plot, assuming the distribution is correct. b) Deviance residuals versus fitted values (on the link scale).

QQ-plotting code from the gratia package (Simpson, 2018), using the qq_plot() function, as this function creates a ggplot2 object that are easier to customize than the base plots from gam. check(). The code for generating these plots is in the supplemental material. These plots (Fig. 14) indicate that the Gamma distribution seems to fit the observed data well except at low values, where the deviance residuals are larger than predicted by the theoretical quantiles (Fig. 14a). There also does not seem to be a pattern in the residual versus fitted values (Fig. 14b), except for a line of residuals at the lowest values, which correspond to all of those observations where a given taxon was absent from the sample.

The k. check () test (Table 2) shows that the default maximum degrees of freedom for the smoothers used in model $I$ are sufficient for all species, as the effective degrees of freedom (EDF) for all estimated smoothers are well below their maximum possible value (k'), and the p-value for the observed k-index (which measures pattern in the residuals) is not significant.

In this table, each row corresponds to a single smooth term, k' corresponds to the number of basis functions used for that smoother in the fitted model (smaller than the specified $\mathrm{k}$ in the model itself, as some basis functions are automatically dropped to ensure the model is identifiable). The column EDF is the estimated Effective Degrees of Freedom for that smoother, the k-index is a measure of the remaining pattern in the residuals, and the p-value is calculated based on the distribution of the k-index after randomizing the order of the residuals. Note that there is no p-value for the random effects smoothers $s$ (taxon) and $\mathbf{s}($ taxon, year_f) as the p-value is calculated from simulation-based tests for autocorrelation of the residuals. As taxon and year_f are treated as simple random effects with no natural ordering, there is no meaningful way of checking for autocorrelation.

Differences between models $S$ (shared smoothness between taxa) and $I$ (different smoothness for each taxa) seem to be driven by the low seasonality of $L$. siciloides relative to the other 
Table 2: Results from running $\mathrm{k}$. check() on zoo_comm_modI. Each row corresponds to a single model term. The notation for term names uses mgcv syntax. For instance, "s(day):taxonC. sphaericus" refers to the the smoother for day for the taxon $C$. sphaericus.

\begin{tabular}{lrrrr}
\hline Model term & k' & EDF & k-index & p-value \\
\hline s(day):taxonC. sphaericus & 8 & 4.78 & 0.89 & 0.44 \\
s(day):taxonCalanoid copepods & 8 & 6.66 & 0.89 & 0.46 \\
s(day):taxonCyclopoid copepods & 8 & 5.31 & 0.89 & 0.46 \\
s(day):taxonD. mendotae & 8 & 6.95 & 0.89 & 0.46 \\
s(day):taxonD. thomasi & 8 & 6.57 & 0.89 & 0.45 \\
s(day):taxonK. cochlearis & 8 & 5.92 & 0.89 & 0.47 \\
s(day):taxonL. siciloides & 8 & 0.52 & 0.89 & 0.46 \\
s(day):taxonM. edax & 8 & 4.69 & 0.89 & 0.43 \\
s(taxon) & 8 & 6.26 & $\mathrm{NA}$ & $\mathrm{NA}$ \\
s(taxon,year_f) & 152 & 51.73 & $\mathrm{NA}$ & $\mathrm{NA}$ \\
\hline
\end{tabular}

species, and how this is captured by the more flexible model $I$ (Fig. 15). Still, both models show very similar fits to the training data. This implies that the added complexity of different penalties for each species (model $I$ ) is unnecessary here, which is consistent with the fact that model $S$ has a lower AIC (4667) than model $I$ (4677), and that model $S$ is somewhat better at predicting out-of-sample fits for all taxa than model $I$ (Table 3). Both models show significant predictive improvement compared to the intercept-only model for all species except $K$. cochlearis (Table 3). This may be driven by changing timing of the spring bloom for this species between training and out-of-sample years (Fig. 15).

Next, we look at how to fit inter-lake variability in dynamics for just Daphnia mendotae. Here, we will compare models $G, G S$, and GI to determine if a single global function is appropriate for all four lakes, or if we can more effectively model variation between lakes with a shared smoother and lake-specific smoothers.

\section{Model $G$ :}

zoo_daph_modG <- gam(density_adj s(day, bs="cc", k=10) + s(lake, bs="re") + s(lake, year_f, bs="re"), data $=$ daphnia_train, knots $=1$ ist $(\operatorname{day}=c(0,365))$, family=Gamma (link="log"), method="REML", drop. unused . levels=FALSE)

\section{Model GS:}

zoo_daph_modGS <- gam(density_adj s(day, bs="cc", k=10) + $\mathrm{s}($ day, lake, $\mathrm{k}=10, \mathrm{bs}=" \mathrm{f} \mathrm{s} ", \mathrm{xt}=\mathrm{list}(\mathrm{bs}=" \mathrm{cc} "))+$ s(lake, year_f, bs="re"), 
Model S Model I

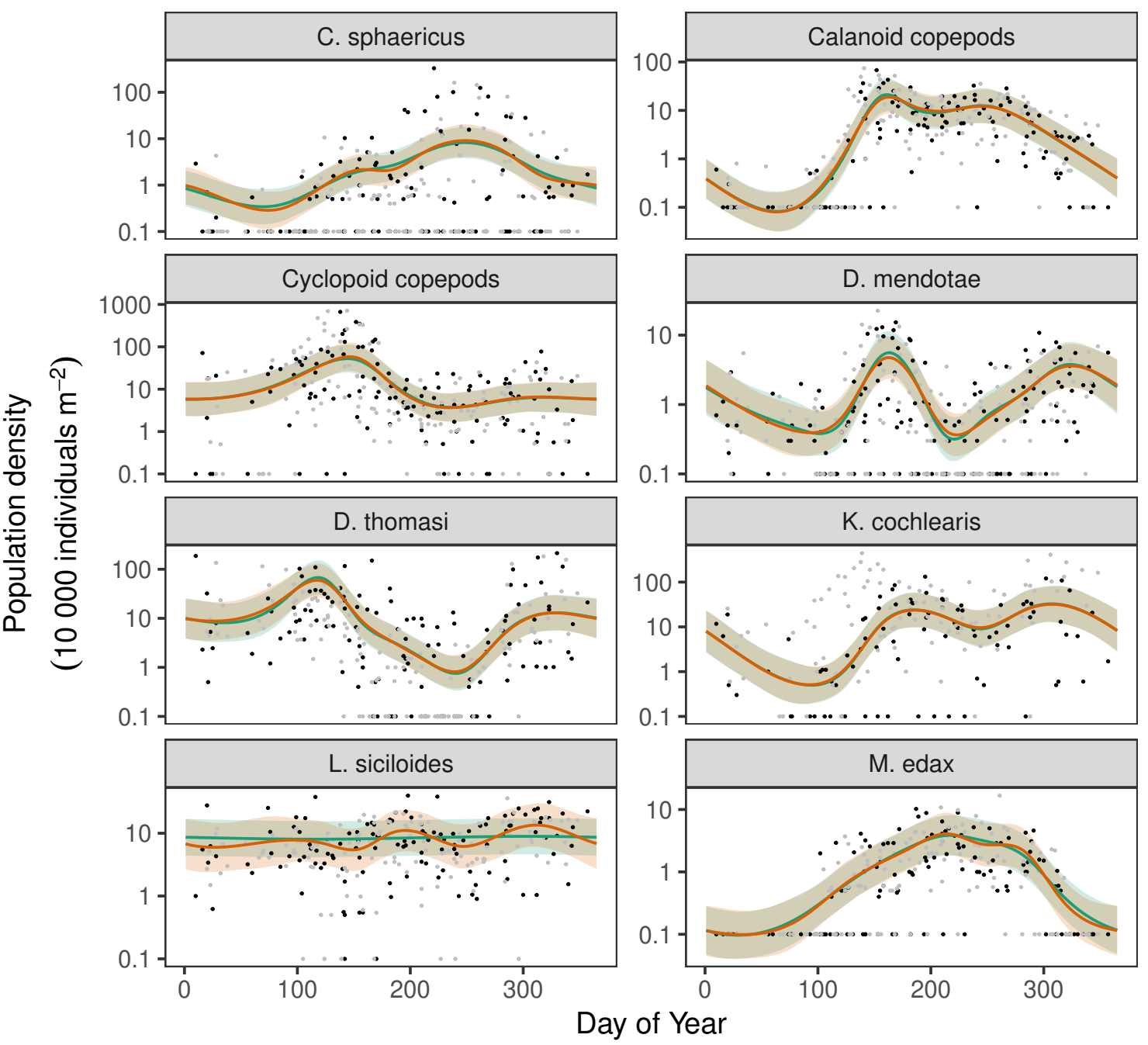

Figure 15: Species-specific seasonal dynamics for the eight zooplankon species tracked in Lake Mendota. Black points indicate individual plankton observations in the training data, and grey points are observations in held-out years used for model validation. Lines indicate predicted average values for model $S$ (green) and model $I$ (red). Ribbons indicate \pm 2 standard errors around the mean. 
Table 3: Out-of-sample predictive ability for model $S$ and $I$ applied to the zooplankton community dataset. Deviance values represent the total deviance of model predictions from observations for out-of-sample data. 'Intercept only' results are for a null model with only taxon-level random effect intercepts included.

\begin{tabular}{llll}
\hline \multirow{2}{*}{ Taxon } & \multicolumn{3}{c}{ Total deviance of out-of-sample data } \\
\cline { 2 - 4 } & Intercept only & Model S & Model I \\
\hline C. sphaericus & 715 & 482 & 495 \\
Calanoid copepods & 346 & 220 & 223 \\
Cyclopoid copepods & 569 & 381 & 386 \\
D. mendotae & 353 & 264 & 268 \\
D. thomasi & 486 & 333 & 337 \\
K. cochlearis & 486 & 2260 & 2340 \\
L. siciloides & 132 & 116 & 126 \\
M. edax & 270 & 138 & 139 \\
\hline
\end{tabular}

data=daphnia_train, knots=list $(\operatorname{day}=c(0,365))$, family=Gamma (link="log"), method="REML", drop . unused . levels=FALSE)

\section{Model GI:}

zoo_daph_modGI <- gam(density_adj s(day, bs="cc", k=10) + s(lake, bs="re") + $\mathrm{s}$ (day, by=lake, $\mathrm{k}=10, \mathrm{bs}=" \mathrm{cc} ")+$ s(lake, year_f, bs="re"),

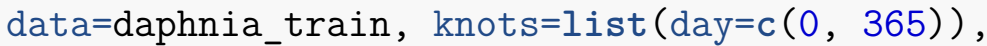
family=Gamma (link ="log"), method="REML", drop . unused . levels=FALSE)

Diagnostic plots from gam. check() indicate that there are no substantial patterns comparing residuals to fitted values (not shown), and QQ-plots are similar to those from the zooplankton community models; the residuals for all three models closely correspond to the expected (Gamma) distribution, except at small values, where the observed residuals are generally larger than expected (Fig. 16). As with the community data, this is likely an artifact of the assumption we made of assigning zero observations a value of 1000 (the lowest possible value), imposing an artificial lower bound on the observed counts. There was also some evidence that the largest observed values were smaller than expected given the theoretical distribution , but these fell within the $95 \%$ CI for expected deviations from the 1-1 line (Fig. 16).

AIC values indicate that both model GS (1093.71) and GI (1085.7) are better fits than model $G$ (1097.62), with model GI fitting somewhat better than model $G S .^{7}$ There does not seem to

\footnotetext{
${ }^{7}$ When comparing models via AIC, we use the standard rule of thumb from Burnham \& Anderson (1998), where models that differ by 2 units or less from the lowest AIC model have substantial support, and those
} 

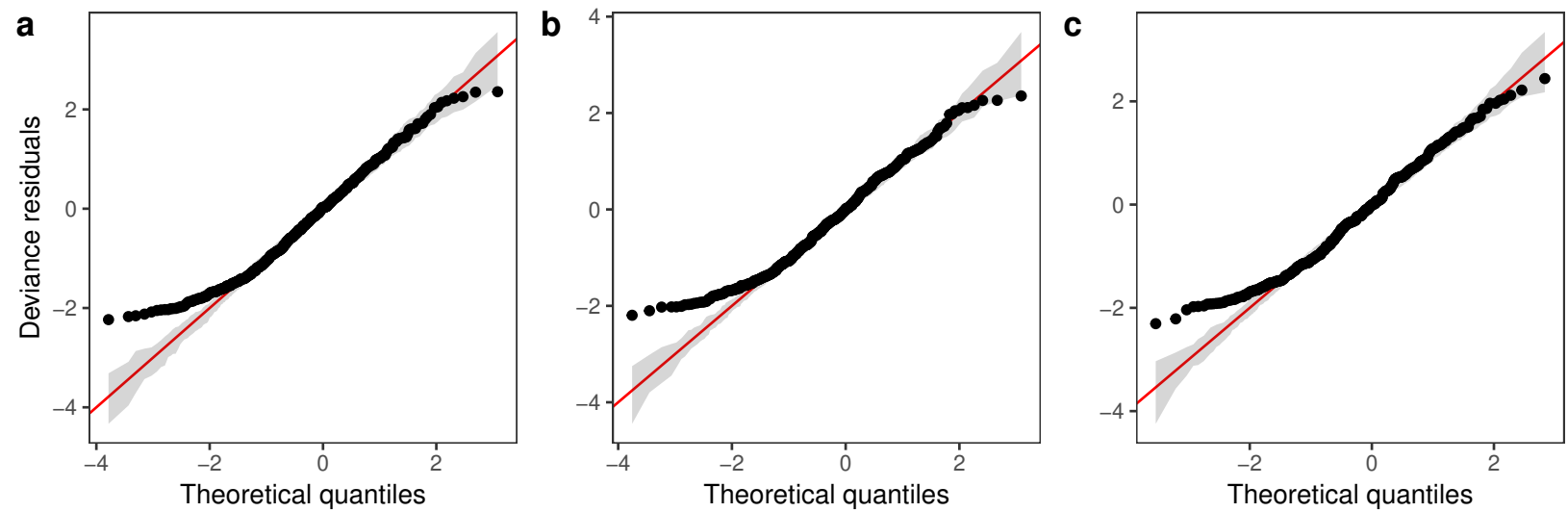

Figure 16: QQ-plots for model $G(\mathrm{a}), G S(\mathrm{~b})$, and $G I$ (c) fitted to Daphnia data across the four lakes. Red line indicates the 1-1 line, black points are observed model residuals, and grey bands correspond to the expected 95\% CI for the QQ plot, assuming the distribution is correct.

be a large amount of inter-lake variability (the effective degrees of freedom per lake are low in models GS \& GI). Plots for all three models (Fig. 17) show that Mendota, Monona, and Kegonsa lakes are very close to the average and to one another for both models, but Waubesa shows evidence of a more pronounced spring bloom and lower winter abundances.

Model $G I$ is able to predict as well or better than model $G$ or $G S$ for all lakes (Table 4), indicating that allowing for inter-lake variation in seasonal dynamics improved model prediction. All three models predicted dynamics in Lake Mendota and Lake Menona significantly better than the intercept-only model (Table 4). None of the models did well in terms of predicting Lake Waubesa dynamics out-of-sample compared to a simple model with only a lake-specific intercept and no intra-annual variability, but this was due to the influence of a single large outlier in the out-of-sample data that occurred after the spring bloom, at day 243 (Fig. 17; note that the y-axis is log-scaled). However, baring a more detailed investigation into the cause of this large value, we cannot arbitrarily exclude this outlier from the goodness-of-fit analysis; it may be due either to measurement error or a true high late-season Daphnia density that our model was not able to predict.

differing by more than 4 units have less support. 


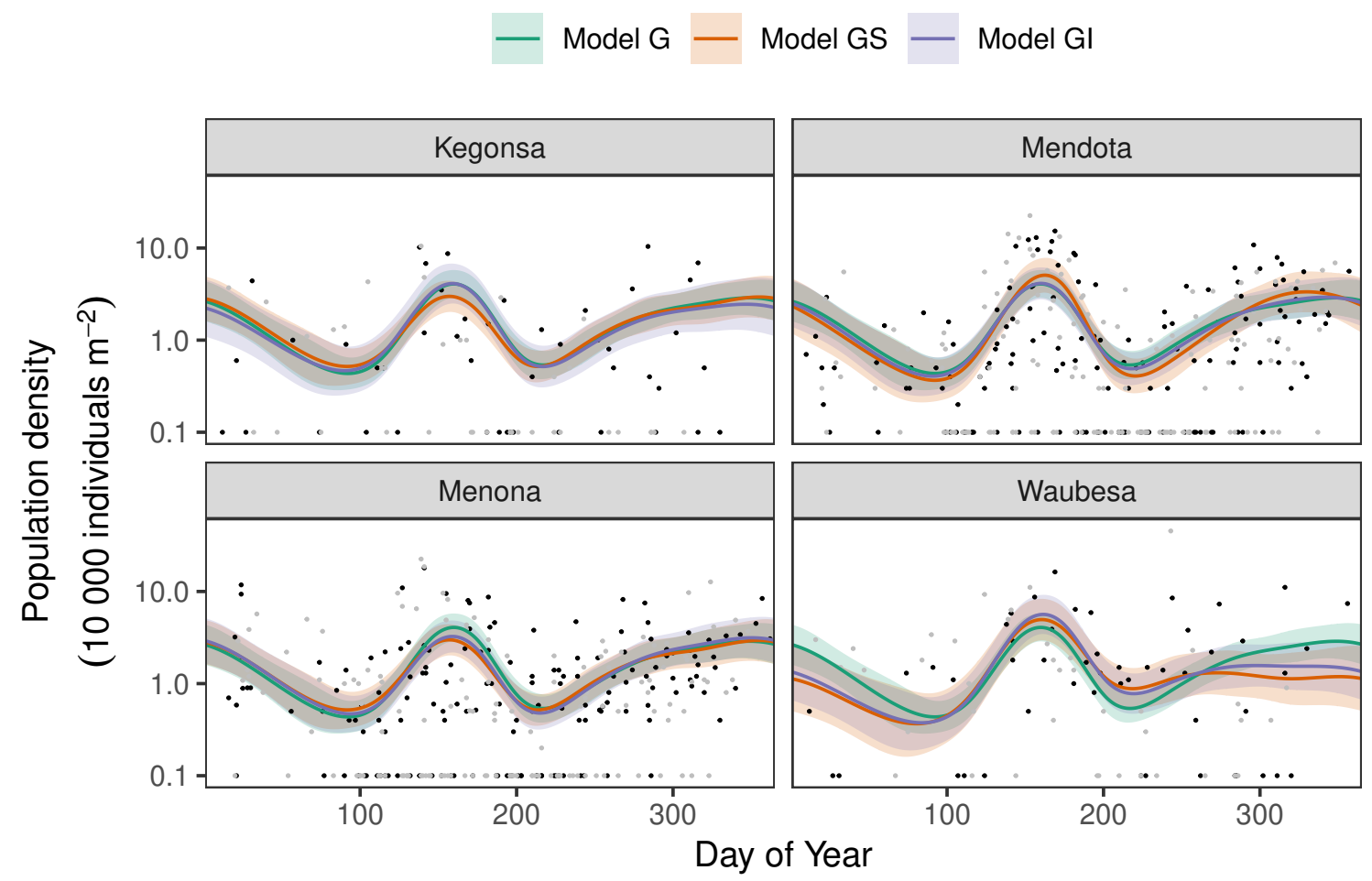

Figure 17: Raw data (points) and fitted models (lines) for D. mendota data. Black points indicate individual plankton observations in the training data, and grey points are observations in held-out years used for model validation. Green line: model $G$ (no inter-lake variation in dynamics); orange line: model $G S$ (interlake variation with similar smoothness); purple line: model GI (varying smoothness among lakes). Shaded bands are drawn at \pm 2 standard errors around each model. 
Table 4: Out-of-sample predictive ability for model G, GS, and GI applied to the D. mendotae dataset. Deviance values represent the total deviance of model predictions from observations for out-of-sample data. 'Intercept only' results are for a null model with only lake-level random effect intercepts included.

\begin{tabular}{lllll}
\hline & \multicolumn{4}{c}{ Total deviance of out-of-sample data } \\
\cline { 2 - 5 } Lake & Intercept only & Model G & Model GS & Model GI \\
\hline Kegonsa & 96 & 92 & 89 & 86 \\
Mendota & 352 & 258 & 257 & 257 \\
Menona & 348 & 300 & 294 & 290 \\
Waubesa & 113 & 176 & 164 & 157 \\
\hline
\end{tabular}

\section{V: Computational and statistical issues when fitting HGAMs}

Which of the five model formulations (Fig. 4) should you choose for a given data set? There are two major trade-offs to consider. The first is the bias-variance trade-off: more complex models can account for more fluctuations in the data, but also tend to give more variable predictions, and can overfit. The second trade-off is model complexity versus computational cost: more complex models can include more potential sources of variation and give more information about a given data set, but will generally take more time and computational resources to fit and debug. We discuss both of these trade-offs in this section. We also discuss how to extend the HGAM framework to fit more complex models.

\section{Bias-variance trade-offs}

The bias-variance trade-off is a fundamental concept in statistics. When trying to estimate any relationship (in the case of GAMs, a smooth relationship between predictors and data) bias measures how far, on average, an estimate is from the true value. The variance of an estimator corresponds to how much that estimator would fluctuate if applied to multiple different samples of the same size taken from the same population. These two properties tend to be traded off when fitting models. For instance, rather than estimating a population mean from data, we could simply use a predetermined fixed value regardless of the observed data ${ }^{8}$. This estimate would have no variance (as it is always the same regardless of what the data look like) but would have high bias unless the true population mean happened to equal the fixed value we chose. Penalization is useful because using a penalty term slightly increases model bias, but can substantially decrease variance (Efron \& Morris, 1977).

In GAMs, the bias-variance trade-off is managed by the terms of the penalty matrix, and equivalently random effect variances in HGLMs. Larger penalties correspond to lower variance, as the estimated function is unable to wiggle a great deal, but also correspond to higher bias unless the true function is close to the null space for a given smoother (e.g., a straight line

\footnotetext{
${ }^{8}$ While this example may seem contrived, this is exactly what happens when we assume a given regression coefficient is equal to zero (and thus exclude it from a model).
} 
for TPRS with 2nd derivative penalties, or zero for a random effect). The computational machinery used by mgcv to fit smooth terms is designed to find penalty terms that best trade-off bias for variance to find a smoother that can effectively predict new data.

The bias-variance trade-off comes into play with HGAMs when choosing whether to fit separate penalties for each group level or assign a common penalty for all group levels (i.e., deciding between models $G S \& G I$ or models $S \& I$ ). If the functional relationships we are trying to estimate for different group levels actually vary in how wiggly they are, setting the penalty for all group-level smoothers equal (models $G S \& S$ ) will either lead to overly variable estimates for the least variable group levels, over-smoothed (biased) estimates for the most wiggly terms, or a mixture of these two, depending on the fitting criteria.

We developed a simple numerical experiment to determine whether mgcv's fitting criteria tend to set estimated smoothness penalties high or low in the presence of among-group variability in smoothness when fitting model $G S$ or $S$ HGAMs. We simulated data from four different groups, with all groups having the same levels of the covariate $x$, equally spaced across the range from 0 to $2 \pi$. For each group, the true function relating $x$ to the response, $y$, was a cosine wave, but the frequency varied from 0.5 (equal to half a cycle across the range of $x$ ) to 4 (corresponding to 4 full cycles across the range). As all four sine waves spanned the whole range from -1 to +1 across the range of $\mathrm{x}$, and as they were all integer or half-integer frequencies, the signal for all groups had the same variance across the range of $x$, approximately equal to 0.5. Therefore, the true function for all groups had the same strength of signal; all that varied between groups was how rapidly the signal fluctuated. We added normally distributed error to all $y$-values, with three different noise levels, given by standard deviations of $0.5,1$, and 2 . These correspond to signal-to-noise ratios (i.e. variance of the cosine curve divided by variance of the noise) of $2,0.5$, and 0.125 . For each noise level we created 25 replicate data sets to illustrate the amount of simulation-to-simulation variation in model fit. We then fit both model $S$ (where all curves were assumed to be equally smooth) and model $I$ (with varying smoothness) to each replicate for each noise level, using REML criteria to estimate penalties.

A sample of the fits for each group for three of the replicates for each model are shown in Fig. 18a, with model $S$ in red and model $I$ in blue. Figure 18b illustrates how well each model fared across the range of replicates at accurately estimating the true smoothness of the highest frequency terms as measured by the squared second derivative of the smooth fit versus that of the true function, with the distance to the black one-to-one line indicating the degree to which the estimated function for each group over- or under-estimated the smoothness of the true signal. In general, under low noise conditions (Fig. 18, signal-to-noise ratio of 2), model $S$ tended to overfit the smoothest, lowest-frequency, groups, while accurately fitting the highest frequency groups. Under moderate signal-to-noise ratios, model $S$ tended to over-penalize high-frequency groups and under-penalize low frequency groups, and in the lowest signal-to-noise ratio tested (0.125), model $S$ tended to penalize all groups towards very smooth functions (Fig. 18b). Curves estimated by model $I$, on the other hand, tended to accurately capture the true wiggliness of the function across the whole range of frequencies and noises, except for the lowest-frequency groups, and the highest frequency groups it the presence of high noise; in both cases, model $I$ tended to over-smooth (Fig. 18b). 
This implies that assuming equal smoothness will result in underestimating the true smoothness of low-variability terms in cases of high signal-to-noise, and overestimating the true smoothness of high-frequency terms in low signal-to-noise data sets. If this is a potential issue, we recommend fitting both models $S$ and $I$ and using standard model evaluation criteria (e.g., AIC) or out-of-sample predictive accuracy (as in Section IV) to determine if there is evidence for among-group variability in smoothness. However, it may be the case that there are too few data points per group to estimate separate smoothness levels, in which case model $G S$ or model $S$ may still be the better option even in the face of varying smoothness.

The ideal case would be to assume that among-group penalties follow their own distribution (estimated from the data), to allow variation in smoothness while still getting the benefit of pooling information on smoothness between groups. This is currently not implemented in mgcv. It is possible to set up this type of varying penalty model in flexible Bayesian modelling software such as Stan (see below for a discussion of how to fit HGAMs using these tools), where inter-group variation in smoothing penalties could be modelled with a hierarchical prior. However, to the best of our knowledge, how to fit this type of model has not been well studied in either the Bayesian or frequentist literature.

It may seem there is also a bias-variance trade-off between choosing to use a single global smoother (model $G$ ) or a global smoother plus group-level terms (models $G S$ and $G I$ ). In model $G$, all the data is used to estimate a single smooth term, and thus should have lower variance than models $G S$ and $G I$, but higher bias for any given group in the presence of inter-group functional variability. However, in practice, this trade-off will be handled via penalization; if there are no average differences between functional responses, mgcv will penalize the group-specific functions toward zero, and thus toward the global model. The choice between using model $G$ versus models $G S$ and $G I$ should generally be driven by computational costs. Model $G$ is typically much faster to fit than models $G S$ and $G I$, even in the absence of among-group differences. If there is no need to estimate inter-group variability, model $G$ will typically be more efficient.

A similar issue exists when choosing between models $G S$ and $G I$ and models $S$ and $I$. If all group levels have very different functional shapes, the global term will get penalized toward zero in models $G S$ and $G I$, so they will reduce to models $S$ and $I$. The choice to include a global term should be made based on scientific considerations (is the global term of interest?) and computational considerations.

\section{Complexity-computation trade-offs}

The more flexible a model is, the larger an effective parameter space any fitting software has to search. It can be surprisingly easy to use massive computational resources trying to fit models to even small datasets. While we typically want to select models based on their fit and our inferential goals, computing resources can often act as an effective upper bound on model complexity. For a given data set, assuming a fixed family and link function, the time taken to estimate an HGAM will depend (roughly) on four factors: (i) the number of coefficients to be estimated (and thus the number of basis functions chosen), (ii) the number of smoothing parameters to be estimated, (iii) whether the model needs to estimate both a 
a

- true value - model S fit - model I fit
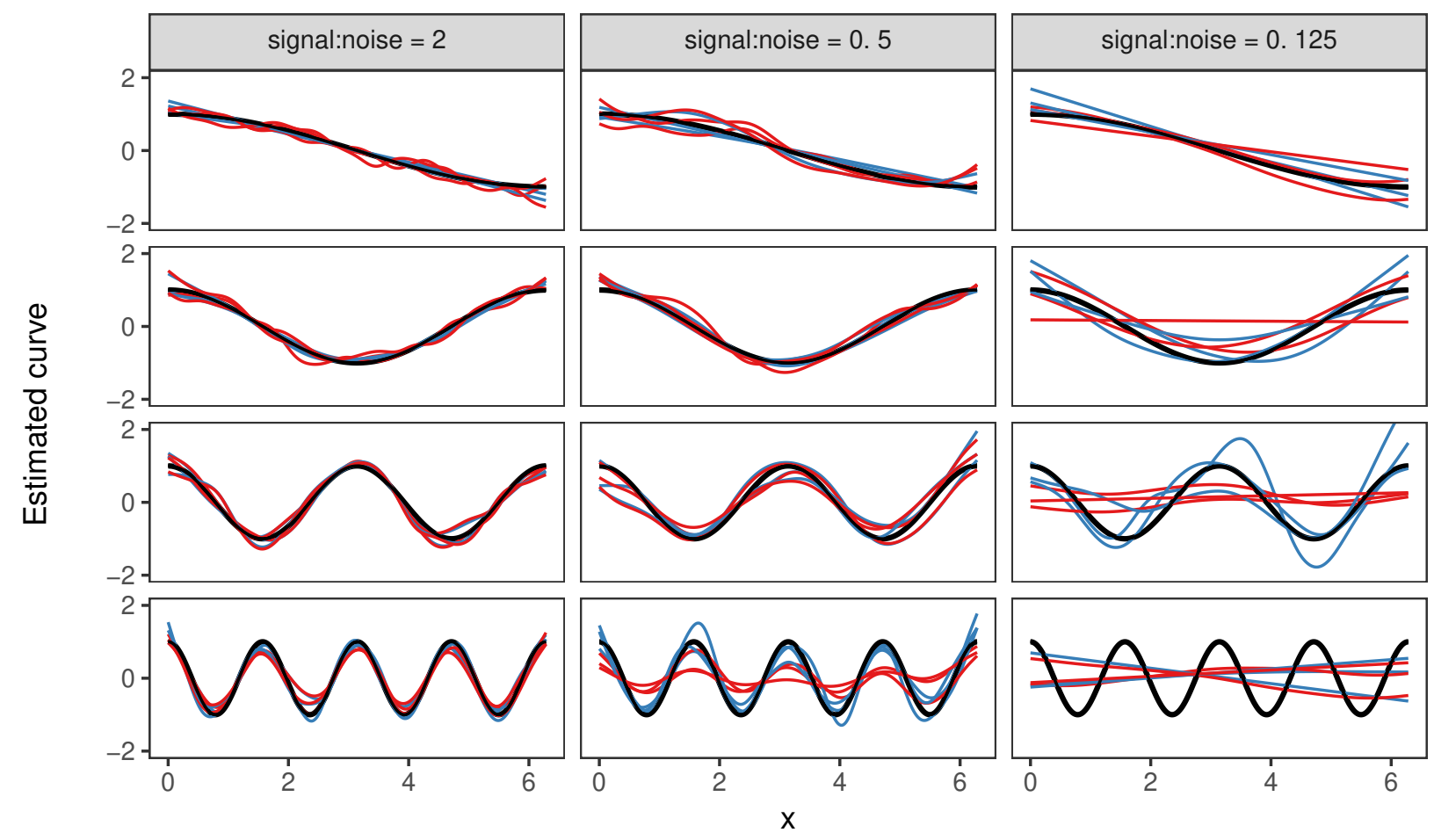

b

- model S fit model I fit
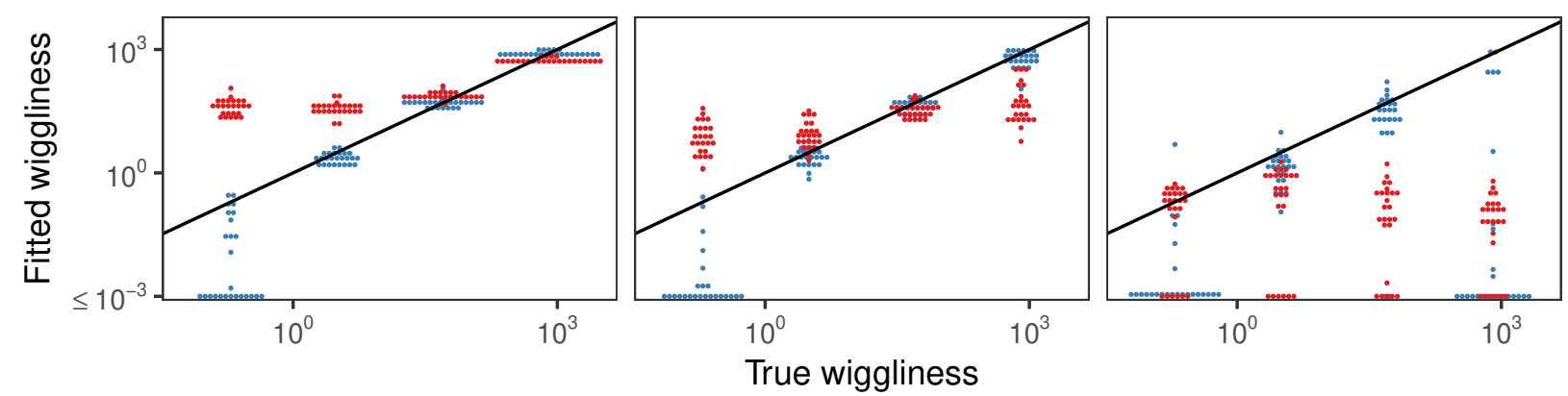

Figure 18: a) Illustration of bias that can arise from assuming equal smoothness for all group levels (model $S$, red lines) versus allowing for intergroup variation in smoothness (model $I$, blue lines) across a range of signal-to-noise ratios, holding the group-level signals constant. The true function for each group level is shown in black. b) Distribution of wiggliness (as measured by the integral of the squared 2nd derivative) of the estimated function for each replicate for each group level for model $S$ (red) and model $I$ (blue), versus the true wiggliness of the function for that grouping level, with the black line indicating the one-to-one line. Points below (above) the black line indicate that a given model estimated the curve as less (more) wiggly than the true curve used to generate the data. Estimated wiggliness less than $10^{-3}$ was truncated for visual clarity, as $\mathbf{m g c v}$ estimated effectively straight lines for several groups, corresponding to a wiggliness of 0 , which would not appear on a log-scaled plot. 
global smoother and group-level smoothers, and (iv) the algorithm and fitting criteria used to estimate parameters.

The most straightforward factor that will affect the amount of computational resources is the number of parameters in the model. Adding group-level smoothers (moving from model $G$ to the other models) means that there will be more regression parameters to estimate. For a dataset with $g$ different groups and $n$ data points, fitting a model with just a global smoother, $\mathrm{y} \sim \mathrm{s}(\mathrm{x}, \mathrm{k}=\mathrm{k})$ will require $k$ coefficients, and takes $\mathcal{O}\left(n k^{2}\right)$ operations to evaluate. Fitting the same data using a group-level smoother (model $S, \mathrm{y} \sim \mathrm{s}(\mathrm{x}, \mathrm{fac}, \mathrm{bs}=" \mathrm{fs} ", \mathrm{k}=\mathrm{k})$ ) will require $\mathcal{O}\left(n k^{2} g^{2}\right)$ operations to evaluate. In effect, adding a group-level smoother will increase computational cost by an order of the number of groups squared. The effect of this is visible in the examples we fit in section III. Table 5 compares the relative time it takes to compute model $G$ versus the other models.

One way to deal with this issue would be to reduce the number of basis functions used when fitting group-level smoothers when the number of groups is large, limiting the flexibility of the model. It can also make sense to use more computationally-efficient basis functions when fitting large data sets, such as P-splines (Wood, 2017b) or cubic splines. TPRSs entail greater computational costs (Wood, 2017a).

Including a global smoother (models $G S$ and $G I$ compared to models $S$ and $I$ ) will not generally substantially affect the number of coefficients that need to be estimated (Table 5). Adding a global term will add at most $\mathrm{k}$ extra terms. It can be substantially less than that, as mgcv drops basis functions from co-linear smoothers to ensure that the model matrix is full rank.

Adding additional smoothing parameters (moving from model $G S$ to $G I$, or moving from model $S$ to $I$ ) is more costly than increasing the number of coefficients to estimate, as estimating smoothing parameters is computationally intensive (Wood, 2011). This means that models $G S$ and $S$ will generally be substantially faster than $G I$ and $I$ when the number of groups is large, as models GI and $I$ fit a separate set of penalties for each group level. The effect of this is visible in comparing the time it takes to fit model GS to model GI (which has a smoother for each group) or models $S$ and $I$ for the CO2 example data (Table 5). Note that this will not hold in all cases. For instance, model $G I$ and $I$ take less time to fit the bird movement data than models $G S$ or $S$ do (Table 5B).

\section{Alternative formulations: $\operatorname{bam}(), \operatorname{gamm}()$, and $\operatorname{gamm} 4()$}

When fitting models with large numbers of groups, it is often possible to speed up computation substantially by using one of the alternative fitting routines available through mgcv.

The first option is the function bam(), which requires the fewest changes to existing code written using the gam() function. bam() is designed to improve performance when fitting large data sets via two mechanisms. First, it saves on memory needed to compute a given model by using a random subset of the data to calculate the basis functions. It then blocks the data and updates model fit within each block (Wood, Goude \& Shaw, 2015). While this is primarily designed to reduce memory usage, it can also substantially reduce computation 
Table 5: Relative computational time and model complexity for different HGAM formulations of the two example data sets from section III. All times are scaled relative to the length of time model $G$ takes to fit to that data set. The number of coefficients measures the total number of model parameters (including intercepts). The number of smoothers is the total number of unique penalty values estimated by the model.

\begin{tabular}{lrrr}
\hline & & \multicolumn{2}{c}{ Number of terms } \\
\cline { 3 - 4 } Model & Relative Time & Coefficients & Penalties \\
\hline A. CO2 data & & & \\
G & 1 & 17 & 2 \\
GS & 7 & 65 & 3 \\
GI & 14 & 65 & 14 \\
S & 5 & 61 & 3 \\
I & 16 & 61 & 13 \\
B. Bird movement data & & \\
G & 1 & 90 & 2 \\
GS & 510 & 540 & 8 \\
GI & 390 & 624 & 14 \\
S & 820 & 541 & 6 \\
I & 70 & 535 & 12 \\
\hline
\end{tabular}

time. Second, when using bam()'s default method="fREML" ("Fast REML") method, you can use the discrete=TRUE option: this first bins continuous covariates into a smaller number of discrete values before estimating the model, substantially reducing the amount of computation needed (Wood et al. (2017); see ?mgcv: : bam for more details). Setting up the five model types (Fig. 4) in bam () uses the same code as we have previously covered; the only difference is that you use the bam() instead of gam() function, and have the additional option of discretizing your covariates.

bam() has a larger computational overhead than gam(); for small numbers of groups it can be slower than gam() (Fig. 19). As the number of groups increases, computational time for bam() increases more slowly than for gam(); in our simulation tests, when the number of groups is greater than 16, bam() can be upward of an order of magnitude faster (Fig. 19). Note that $\operatorname{bam}()$ can be somewhat less computationally stable when estimating these models (i.e., less likely to converge). While base bam() (not fit using discrete=TRUE) is slower than the other approaches shown in Fig. 19, that does not imply that bam() is a worse choice in general; it is designed to avoid memory limitations when working with big data rather than explicitly speeding up model fitting. The bam() functions would likely show much better relative performance when the number of individuals per group were large (in the hundreds to thousands, compared to the 20 individuals per group used in Fig. 19).

The second option is to fit models using one of two dedicated mixed effect model estimation packages, nlme and lme4. The mgcv package includes the function gamm(), which uses the nlme package to estimate the GAM, automatically handling the transformation of smooth 
terms into random effects (and back into basis function representations for plotting and other statistical analyses). The gamm4() function, in the separate gamm4 package, uses lme4 in a similar way. Using gamm() or gamm4() to fit models rather than gam() can substantially speed up computation when the number of groups is large, as both nlme and lme4 take advantage of the sparse structure of the random effects, where most basis functions will be zero for most groups (i.e., any group-specific basis function will only take a non-zero value for observations in that group level). As with bam(), gamm() and gamm4() are generally slower than gam() for fitting HGAMs when the number of group levels is small (in our simulations, $<8$ group levels), however they do show substantial speed improvements even with a moderate number of groups, and were as fast as or faster to calculate than bam() for all numbers of grouping levels we tested (Fig. 19) ${ }^{9}$.

Both gamm() and gamm4() require a few changes to model code. First, there are a few limitations on how you are able to specify the different model types (Fig. 4) in both frameworks. Factor-smoother interaction ( $b s=" f s ")$ basis setup works in both gamm() and gamm4(). However, as the nlme package does not support crossed random effects, it is not possible to have two factor-smoother interaction terms for the same grouping variable in gamm() models (e.g., $y \sim s(x 1, \operatorname{grp}, b s=" f s ")+s(x 2, \operatorname{grp}, b s=" f s ")$. These type of crossed random effects are allowed in gamm4. The use of te() terms are not possible in gamm4, due to issues with how random effects are specified in the lme4 package, making it impossible to code models where multiple penalties apply to a single basis function. Instead, for multidimensional group-level smoothers, the alternate function t2() needs to be used to generate these terms, as it creates tensor products with only a single penalty for each basis function (see ?mgcv: : t2 for details on these smoothers, and Wood, Scheipl \& Faraway (2013) for the theoretical basis behind this type of tensor product). For instance, model $G S$ for the bird movement data we discussed in section III would need to be coded as:

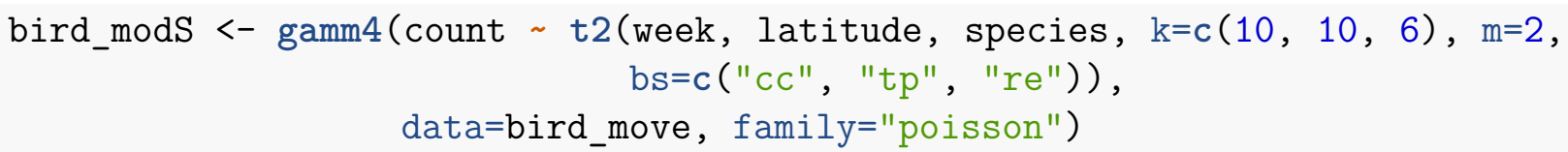

These packages also do not support the same range of families for the dependent variable; gamm() only supports non-Gaussian families by using a fitting method called penalized quasilikelihood (PQL) that is slower and not as numerically stable as the methods used in gam(), bam(), and gamm4(). Non-Gaussian families are well supported by lme4 (and thus gamm4), but can only fit them using marginal likelihood (ML) rather than REML, so may tend to over-smooth relative to gam() using REML estimation. Further, neither gamm() nor gamm4() supports several of the extended families available through $\mathbf{m g c v}$, such as zero-inflated, negative binomial, or ordered categorical and multinomial distributions.

\footnotetext{
${ }^{9}$ It is also possible to speed up both gam() and bam() by using multiple processors in parallel, whereas this is not currently possible for gamm() and gamm4(). For large numbers of grouping levels, this should speed up computation as well, at the cost of using more memory. However, computation time will likely not decline linearly with the number of cores used, since not all model fitting sets are parallelizable, and performance of cores can vary. As parallel processing can be complicated and dependent on the type of computer you are using to configure, we do not go into how to use these methods here. The help file ?mgcv: :mgcv.parallel explains how to use parallel computations for $\operatorname{gam}()$ and bam() in detail.
} 


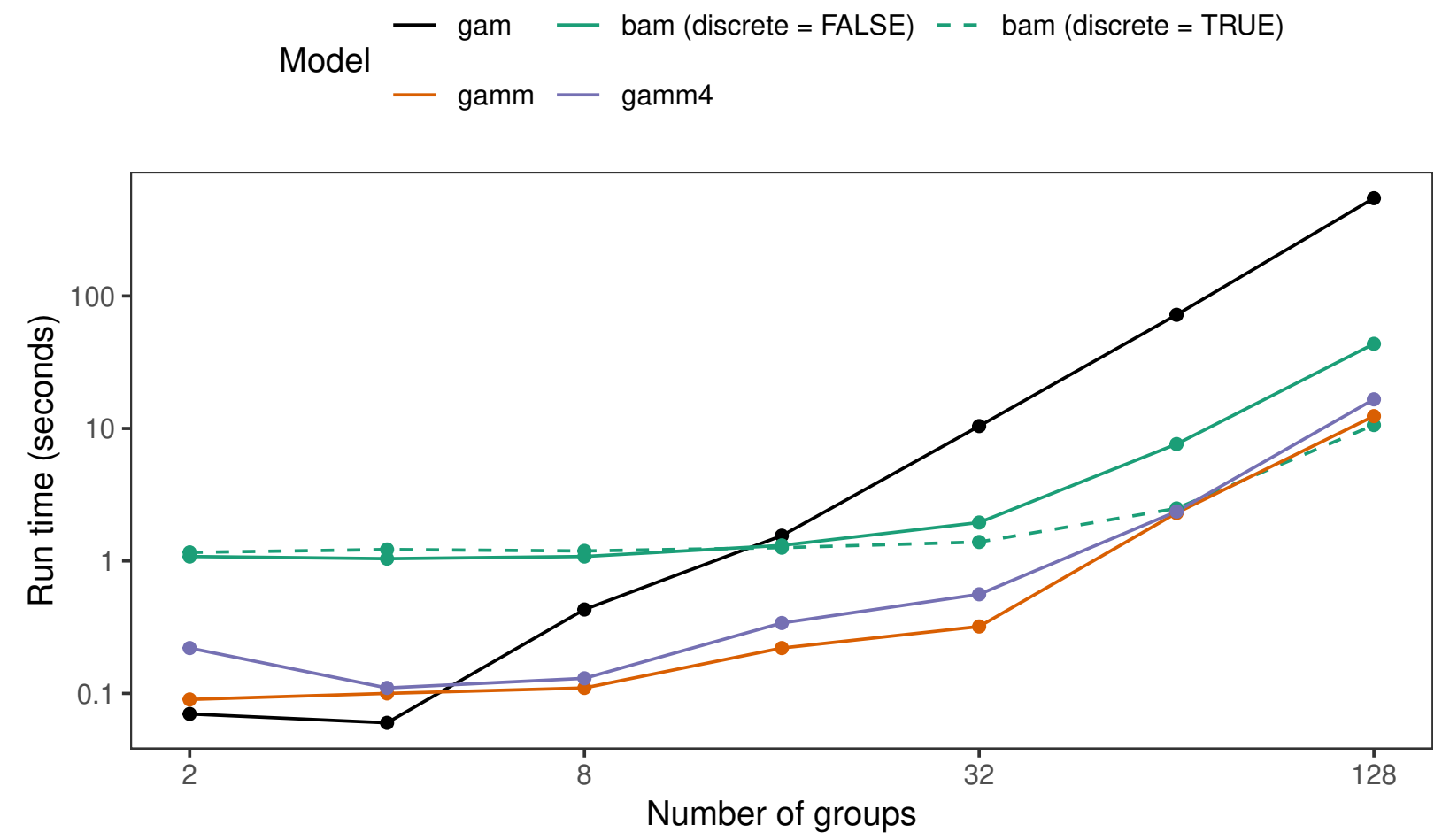

Figure 19: Elapsed time to estimate the same model using each of the four approaches. Each data set was generated with 20 observations per group using a unimodal global function and random group-specific functions consisting of an intercept, a quadratic term, and logistic trend for each group. Observation error was normally distributed. Models were fit using model 2: y $s(x, k=10, b s=" c p ")+s(x, f a c, k=10, b s=" f s ", x t=l i s t(b s=" c p "), m=1)$. All models were run on a single core. 
${ }_{811}$ Estimation issues when fitting both global and group-level

\footnotetext{
${ }^{10}$ There is an important caveat here. When fitting $G S$ models using tensor products in mgcv, the global and group-level terms will not be entirely concurve because $\mathbf{m g c v}$ will automatically drop basis functions from the group-level smoother to ensure that these terms are not perfectly concurve. That is, so that no basis function in the global term could be formed from a linear combination of group-level basis functions (see ?mgcv: : gam. side for how terms to be dropped are selected). Group-level terms fit using bs="fs" smoothers will not have any basis functions dropped, as mgcv disables checking for side-constraints for these smoothers (since all basis functions are fully penalized for this type of smoother, in principle concurvity should not be an issue; see ?mgcv: : smooth.construct.fs.smooth.spec for details).
} 


\footnotetext{
${ }^{11}$ It is also important to consider here that the concept of a "global function" is a bit fuzzy itself, and there are many possible ways to define what a global function is (as we discussed in section III). The global function being fit in all of these models is actually an average function, and the shape of it will depend on the sampling structure of any given study. In our view, the global function fitted in these models should generally be viewed as a useful summary of an average trend across a wide range of groups, and would only represent an actual average relationship if the grouping levels were drawn at random from some underlying population and if there was scientific reason to believe that individual groups should differ from the mean only via some additive function.
} 

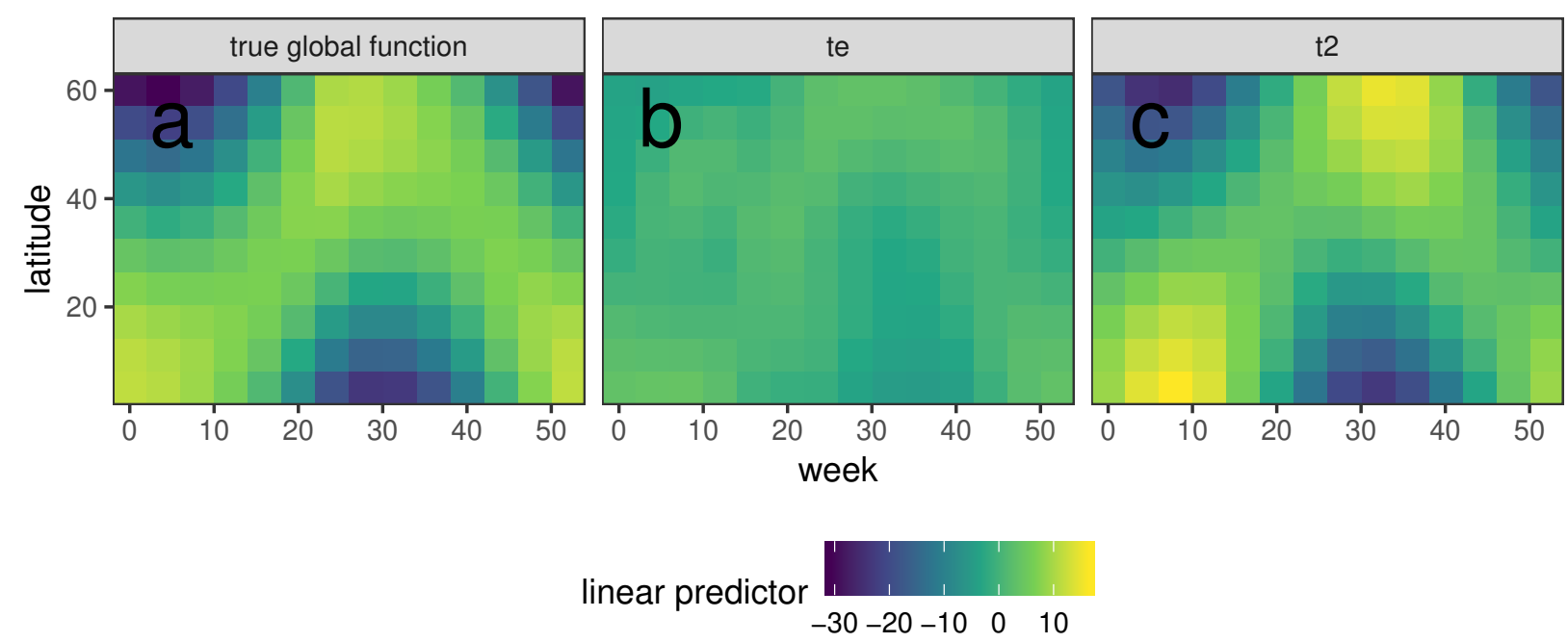

Figure 20: Average global function used for simulating bird_move data set (a) compared to the fitted global function for a $G S$ model estimated with either a te() smoother (b) or a t2() smoother with full=TRUE (c) for group-level terms. Both group-level smoothers used the same model specification as in section III except for the type of tensor product used. Colors indicate the value of the linear predictor of bird density at each location in each week.

considered equally likely). Any terms in the range space are treated as having a multivariate normal prior, and the smoothing parameters are treated as having an improper flat prior (see Wood (2017a) Section 5.8 for more details on this connection). The posterior covariance matrix (Wood, 2006b) for model parameters can be extracted from any fitted gam() or bam() model with vcov(model) (this is conditional on the estimated smoothing parameters unless the option unconditional=TRUE is specified). Given the normal posterior for the estimates of $\boldsymbol{\beta}$, we can sample from a multivariate normal with mean $\hat{\boldsymbol{\beta}}$ and posterior covariance matrix. Such samples can be used to estimate uncertainty in functions of the predictors. Viewing our GAM as Bayesian is a somewhat unavoidable consequence of the equivalence of random effects and splines - if we think that there is some true smoother that we wish to estimate, we must take a Bayesian view of our random effects (splines) as we do not think that the true smoother changes each time we collect data (Wood, 2017a, Section 5.8). The standard confidence intervals used in $\mathbf{m g c v}$ are in fact Bayesian posterior credible intervals, which happen to have good frequentist across-the-function properties (Wood, 2006b; Marra \& Wood, 2012). The newest version of $\mathbf{m g c v}$ as of this writing (v. 1.8-28) also includes an experimental implementation of Integrated Nested Laplace Approximation (INLA) to calculate full posterior distributions for GAMs, via the ginla function (Wood, 2019).

This also means that HGAMs can be included as components in a more complex fully Bayesian model. The mgcv package includes a function jagam() that can take a specified model formula and automatically convert it into code for the JAGS (or BUGS) Bayesian statistical packages, which can be adapted by the user to their own needs. 
Similarly, the brms package (Bürkner, 2017), which can fit complex statistical models using the Bayesian software Stan (Carpenter et al., 2017) allows for the inclusion of $\mathbf{m g c v}$-style smooth terms as part of the model specification. The brms package does not currently support te() tensor products, but does support factor-smooth interactions and t2()-style tensor products, which means all of the models fitted in this paper can be fitted by brms. Finally, the bamlss package (Umlauf, Klein \& Zeileis, 2018) can fit complex GAMs using a number of computational backends, including JAGS and BayesX, using mgcv syntax for model specification.

\section{Beyond HGAMs: functional regression}

The HGAMs we have discussed are actually a type of functional regression, which is an extension of standard regression models to cases where the outcome variable $y_{i}$ and/or the predictor variables $x_{i}$ for a given outcome are functions, rather than single variables (Ramsay \& Silverman, 2005). HGAMs as we have described them are a form of function-on-scalar regression (Ramsay \& Silverman, 2005; Reiss, Huang \& Mennes, 2010), where we are trying to estimate a smooth function that varies between grouping levels. Here the "scalar" refers to the grouping level, and the function is the smooth term that varies between levels; in contrast, a standard GAM is a type of scalar-on-scalar regression, as the goal is to use a set of single values (scalars) to estimate each (scalar) response.

We have deliberately focused our paper on these simpler classes of functional regression model, and chosen to use the term HGAM rather than functional regression, as we believe that this more clearly connects these models to modelling approaches already familiar to ecologists. Further, we consider the unit of analysis to still be individual observations, as compared to functional regression where the unit of analysis is whole functions. For instance, we are interested in applications such as species distribution modelling, where the presence of a given species may be predicted from a sum of several species-specific functions of different environmental variables.

However, there is an extensive literature dedicated to the estimation of more complex functional regression models for any interested reader (see Morris (2015) and Greven \& Scheipl (2017) for a good introduction and overview of more recent work in this field). The refund package (Greven \& Scheipl, 2017) uses the statistical machinery of mgcv to fit these models, and should be usable by anyone familiar with mgcv modelling syntax. Functional regression is also a major area of study in Bayesian statistics (e.g., Kaufman, Sain \& others (2010)).

\section{Conclusion}

HGAMs are a powerful tool to model inter-group variability, and we have attempted to illustrate some of the range and possibilities that these models are capable of, how to fit them, and some issues that may arise during model fitting and testing. Specifying these models and techniques for fitting them are active areas statistical research, so this paper should be viewed as a jumping-off point for these models, rather than an end-point; we refer the reader to the rich literature on GAMs (e.g. Wood, 2017a) and functional regression (Ramsay \& Silverman, 
2005; Kaufman, Sain \& others, 2010; Scheipl, Staicu \& Greven, 2014) for more on these ideas.

\section{Acknowledgements}

The authors would like to thank Carly Ziter, Tiago Marques, Jake Walsh, Geoff Evans, Paul Regular, Laura Wheeland, and Isabella Ghement for their thoughtful feedback on earlier versions of this manuscript, and the Ecological Society of America for hosting the mgcv workshops that this work started from. EJP was funded by National Science and Engineering Research Council of Canada (NSERC) and Fisheries and Oceans Canada. GLS is funded by a NSERC Discovery Grant (RGPIN-2014-04032). DLM was partly funded by OPNAV N45 and the SURTASS LFA Settlement Agreement, managed by the U.S. Navy's Living Marine Resources program under Contract No. N39430-17-C-1982. NMR was partially funded by the USAID PREDICT-2 program.

All authors contributed to developing the initial idea for this paper, and to writing and editing the manuscript. Author order after the first author was chosen using the code:

set.seed (11)

sample(c('Miller' , 'Ross' ', 'Simpson'))

All code used to generate this paper, as well as prior versions of this manuscript, are available at: github.com/eric-pedersen/mixed-effect-gams.

\section{Bibliography}

Baayen RH., Rij J van., Cat C de., Wood S. 2018. Autocorrelated errors in experimental data in the language sciences: some solutions offered by Generalized Additive Mixed Models. In: Mixed-Effects Regression Models in Linguistics. Springer, 49-69.

Bates D., Mächler M., Bolker B., Walker S. 2015. Fitting linear mixed-effects models using lme4. Journal of Statistical Software 67:1-48.

Bolker BM., Brooks ME., Clark CJ., Geange SW., Poulsen JR., Stevens MHH., White J-SS. 2009. Generalized linear mixed models: a practical guide for ecology and evolution. Trends in Ecology \& Evolution 24:127-135.

Burnham KP., Anderson DR. 1998. Model selection and inference: A practical informationtheoretic approach. New York, NY: Springer Science \& Business Media.

Bürkner P-C. 2017. brms: An R package for Bayesian multilevel models using Stan. Journal of Statistical Software 80:1-28.

Carpenter B., Gelman A., Hoffman MD., Lee D., Goodrich B., Betancourt M., Brubaker M., Guo J., Li P., Riddell A. 2017. Stan: A probabilistic programming language. Journal of 
statistical software 76.

de Boor C. 1978. A Practical Guide to Splines. Springer.

Dormann CF., Calabrese JM., Guillera-Arroita G., Matechou E., Bahn V., Bartoń K., Beale CM., Ciuti S., Elith J., Gerstner K., Guelat J., Keil P., Lahoz-Monfort JJ., Pollock LJ., Reineking B., Roberts DR., Schröder B., Thuiller W., Warton DI., Wintle BA., Wood SN., Wüest RO., Hartig F. 2018. Model averaging in ecology: A review of Bayesian, informationtheoretic, and tactical approaches for predictive inference. Ecological Monographs 88:485-504. DOI: $10.1002 /$ ecm.1309.

Efron B., Morris C. 1977. Stein's paradox in statistics. Scientific American 236:119-127.

Forster M., Sober E. 2011. AIC scores as evidence: A Bayesian interpretation. In: Bandyopadhyay PS, Forster MR eds. Philosophy of Statistics. Handbook of the Philosophy of Science. Boston, MA: Elsevier B.V., 535-549.

Gelman A. 2006. Multilevel (hierarchical) modeling: what it can and cannot do. Technometrics 48:432-435.

Gelman A., Carlin J., Stern H., Dunson D., Vehtari A., Rubin D. 2013. Bayesian Data Analysis, third edition. Taylor \& Francis.

Greven S., Scheipl F. 2017. A general framework for functional regression modelling. Statistical Modelling 17:1-35. DOI: 10.1177/1471082X16681317.

Hastie TJ., Tibshirani RJ. 1990. Generalized Additive Models. Taylor \& Francis.

Kaufman CG., Sain SR., others. 2010. Bayesian functional ANOVA modeling using gaussian process prior distributions. Bayesian Analysis 5:123-149.

Kimeldorf GS., Wahba G. 1970. A correspondence between Bayesian estimation on stochastic processes and smoothing by splines. The Annals of Mathematical Statistics 41:495-502. DOI: 10.1214/aoms/1177697089.

Laird NM., Ware JH. 1982. Random-effects models for longitudinal data. Biometrics 38:963-974.

Lathrop RC. 2000. Madison Wisonsin Lakes Zooplankton 1976 - 1994. Environmental Data Initiative.

Marra G., Wood SN. 2011. Practical variable selection for generalized additive models. Computational Statistics \&3 Data Analysis 55:2372-2387. DOI: 10.1016/j.csda.2011.02.004.

Marra G., Wood SN. 2012. Coverage properties of confidence intervals for generalized additive model components. Scandinavian Journal of Statistics 39:53-74. DOI: 10.1111/j.14679469.2011.00760.x.

McCullagh P., Nelder JA. 1989. Generalized Linear Models, Second Edition. CRC Press. McMahon SM., Diez JM. 2007. Scales of association: hierarchical linear models and the 
measurement of ecological systems. Ecology Letters 10:437-452.

Morris JS. 2015. Functional regression. Annual Review of Statistics and Its Application 2:321-359. DOI: 10.1146/annurev-statistics-010814-020413.

Potvin C., Lechowicz M., Tardif S. 1990. The statistical analysis of ecophysiological response curves obtained from experiments involving repeated measures. Ecology:1389-1400.

$\mathrm{R}$ Core Team. 2018. $R$ : A language and environment for statistical computing. Vienna, Austria: R Foundation for Statistical Computing.

Ramsay J., Silverman B. 2005. Functional Data Analysis. New York, NY: Springer Science+Business Media, Inc.

Reiss PT., Huang L., Mennes M. 2010. Fast function-on-scalar regression with penalized basis expansions. The International Journal of Biostatistics 6. DOI: 10.2202/1557-4679.1246.

Ruppert D., Wand MP., Carroll RJ. 2003. Semiparametric Regression. Cambridge University Press.

Scheipl F., Gertheiss J., Greven S. 2016. Generalized functional additive mixed models. Electronic Journal of Statistics 10:1455-1492. DOI: 10.1214/16-EJS1145.

Scheipl F., Staicu A-M., Greven S. 2014. Functional additive mixed models. Journal of Computational and Graphical Statistics 24:477-501.

Simpson GL. 2018. Gratia: Graceful ggplot-based graphics and other useful functions for gams fitted using mgcv.

Umlauf N., Klein N., Zeileis A. 2018. BAMLSS: Bayesian additive models for location, scale, and shape (and beyond). Journal of computational and graphical statistics 27:612-627. DOI: 10.1080/10618600.2017.1407325.

Verbyla AP., Cullis BR., Kenward MG., Welham SJ. 1999. The analysis of designed experiments and longitudinal data by using smoothing splines. Journal of the Royal Statistical Society: Series C (Applied Statistics) 48:269-311. DOI: 10.1111/1467-9876.00154.

Wickham H. 2016. Ggplot2: Elegant graphics for data analysis. Springer-Verlag New York.

Wieling M., Tomaschek F., Arnold D., Tiede M., Bröker F., Thiele S., Wood SN., Baayen RH. 2016. Investigating dialectal differences using articulography. Journal of Phonetics 59:122-143.

Wood SN. 2003. Thin plate regression splines. Journal of the Royal Statistical Society: Series $B$ (Statistical Methodology) 65:95-114.

Wood SN. 2006a. Low-rank scale-invariant tensor product smooths for generalized additive mixed models. Biometrics 62:1025-1036. DOI: 10.1111/j.1541-0420.2006.00574.x.

Wood SN. 2006b. On confidence intervals for generalized additive models based on penalized regression splines. Australian $\&$ New Zealand Journal of Statistics 48:445-464.

Wood SN. 2011. Fast stable restricted maximum likelihood and marginal likelihood estimation 
1043 of semiparametric generalized linear models. Journal of the Royal Statistical Society: Series

1044

1045

1046

1047

1048

1049

1050

1051

1052

1053

1054

1055

1056

1057

1058

1059

1060

1061

1062

B (Statistical Methodology) 73:3-36. DOI: 10.1111/j.1467-9868.2010.00749.x.

Wood SN. 2017a. Generalized Additive Models: An Introduction with R, 2nd Edition. Boco Raton, FL: CRC Press.

Wood SN. 2017b. P-splines with derivative based penalties and tensor product smoothing of unevenly distributed data. Statistics and Computing 27:985-989. DOI: 10.1007/s11222-0169666-x.

Wood S. 2019. Simplified integrated nested laplace approximation. Biometrika In Press.

Wood SN., Goude Y., Shaw S. 2015. Generalized additive models for large data sets. Journal of the Royal Statistical Society: Series C (Applied Statistics) 64:139-155. DOI: 10.1111/rssc. 12068 .

Wood SN., Li Z., Shaddick G., Augustin NH. 2017. Generalized additive models for gigadata: Modeling the U.K. black smoke network daily data. Journal of the American Statistical Association 112:1199-1210. DOI: 10.1080/01621459.2016.1195744.

Wood SN., Pya N., Säfken B. 2016. Smoothing parameter and model selection for general smooth models. Journal of the American Statistical Association 111:1548-1563. DOI: 10.1080/01621459.2016.1180986.

Wood SN., Scheipl F., Faraway JJ. 2013. Straightforward intermediate rank tensor product smoothing in mixed models. Statistics and Computing 23:341-360. DOI: 10.1007/s11222-0129314-z. 